\title{
A Comparative Study on Phasor and Frequency Measurement Techniques in Power Systems
}

\author{
Aravinth Sridharan
}

A Thesis Submitted to

Indian Institute of Technology Hyderabad

In Partial Fulfillment of the Requirements for

The Degree of Master of Technology

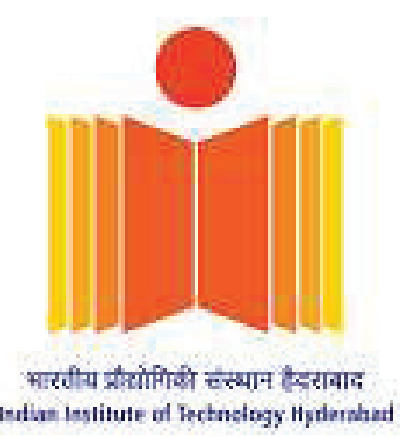

Department of Electrical Engineering

June 2016 


\section{Declaration}

I declare that this written submission represents my ideas in my own words, and where ideas or words of others have been included, I have adequately cited and referenced the original sources. I also declare that I have adhered to all principles of academic honesty and integrity and have not misrepresented or fabricated or falsified any idea/data/fact/source in my submission. I understand that any violation of the above will be a cause for disciplinary action by the Institute and can also evoke penal action from the sources that have thus not been properly cited, or from whom proper permission has not been taken when needed.

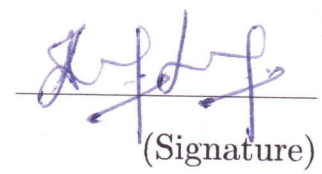

ARAVINIH SRIDHARAN

(Student name)

EEIAMTECHIIO26

(Roll No.) 


\section{Approval Sheet}

This Thesis entitled A Comparative Study on Phasor and Frequency Measurement Techniques in Power Systems by Aravinth Sridharan is approved for the degree of Master of Technology from IIT Hyderabad

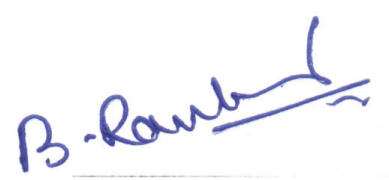

(Dr. B. Ravi Kumar) Examincr Department of Electrical Engincering

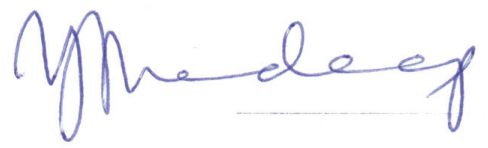

(Dr. Y. Pradecp Kumar) Examiner Department of Electrical Enginecring IIT'II

$$
\text { Vaskar Sarkar }
$$

(I)r. Vaskar Sarkar) Adriscr Department of Electrical Enginesring

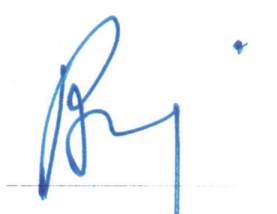

(Dr. Raja Bancrjec) Chairman

Department of Mechanical and Acrospace Enginecring 


\section{Acknowledgements}

I express my deepest gratitude to my project guide Dr. Vaskar Sarkar, Assistant Professor, Department of Electrical Engineering, IIT Hyderabad, whose encouragement, guidance and support from the initial to the final level enabled me to develop an understanding of the subject. It was a great honour for me to pursue my Masters under his supervision.

I am grateful to my committee members Dr. B. Ravi Kumar and Dr. Y. Pradeep Kumar, Assistant Professors, Department of Electrical Engineering, IIT Hyderabad, for their valuable comments and suggestions. I also thank my fellow graduates from Power Electronics and Power Systems stream.

My thanks and appreciations also goes to my classmates and friends who have helped me in developing the project. 


\section{Dedication}

my family 


\begin{abstract}
The objective of the thesis is to make a comparative study on different phasor and frequency estimation algorithms available for power systems. The three-phase power system is designed to operate in a near balanced fashion with the system voltage and current quantities maintained within their nominal values. In practice, that is not always possible. The dynamic nature of the power system signals has made the estimation of these quantities difficult. Over the years, many digital algorithms have been developed to provide accurate real-time frequency and phasor measurements. In this report, four different algorithms for frequency estimation and three different algorithms for phasor estimation are studied and compared. A case study is performed by considering a three-phase voltage signal with time-varying amplitude and frequency to verify the effectiveness of these estimation algorithms.

The concept of time scaling phenomenon is explained in this thesis. The effect of time scaling on the phasors that are measured based on dynamic and static time references are also discussed. A laboratory setup of Phasor Measurement Unit is developed through a Hardware in Loop (HIL) simulation using a real-time simulator based on the DFT algorithms that are considered for the study.
\end{abstract}




\section{Contents}

Declaration ....................................... ii

Approval Sheet . . . . . . . . . . . . . . . . . . . iii

Acknowledgements ................................ iv

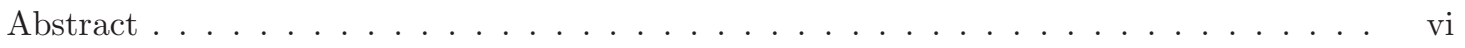

$\begin{array}{lc}\text { Nomenclature } & \text { viii }\end{array}$

1 Introduction $\quad 2$

2 Literature Overview $\quad 5$

2.1 Phasor Estimation Techniques . . . . . . . . . . . . . . . . . . 5

2.1.1 Newton type algorithm . . . . . . . . . . . . . . . . 5

2.1.2 Recursive Gauss-Newton adaptive filter . . . . . . . . . . . . . . . . 5

2.2 Frequency Estimation Techniques . . . . . . . . . . . . . . . . . 6

2.2.1 Zero-Crossing Methods . . . . . . . . . . . . . . . 6

2.2 .2 Discrete Fourier Transform . . . . . . . . . . . . . . . . . . . . . 6

2.2.3 Extended Complex Kalman filter . . . . . . . . . . . . . . . . . . . . 6

2.2 .4 Least Mean Square . . . . . . . . . . . . . . . . . . . . 6

2.2.5 Nonlinear Least Square . . . . . . . . . . . . . . . . . . . 6

3 Phasor Measurement Techniques $\quad 7$

3.1 Concept of Phasor . . . . . . . . . . . . . . . . . . . . . . 7

3.2 Phasor Estimation Techniques . . . . . . . . . . . . . . . . . . . 8

3.2 .1 Discrete Fourier Transform . . . . . . . . . . . . . . . . . . 8

3.2.2 Smart Discrete Fourier Transform . . . . . . . . . . . . . . . . . . . 9

3.2 .3 Phase Locked Loop . . . . . . . . . . . . . . . . . . . . . . . . . . . . . . . 10

3.3 Simulation Results . . . . . . . . . . . . . . . . . . . . . . . . . 11

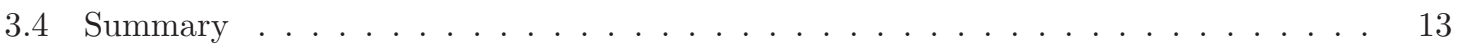

4 Frequency Measurement Techniques $r$

4.1 Complex Valued Least Square . . . . . . . . . . . . . . . . . . . . . . . . . . . 14

4.2 Prony Method . . . . . . . . . . . . . . . . . . . . 16

4.3 Polynomial Fitting . . . . . . . . . . . . . . . . . . . . . . 18

4.4 Simulation Results . . . . . . . . . . . . . . . . . . . . . . . . 19

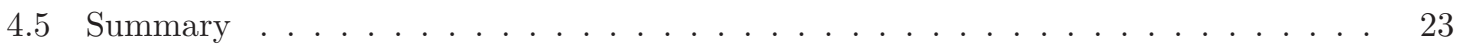


5 Time Scaling Phenomenon in Phasor Calculation

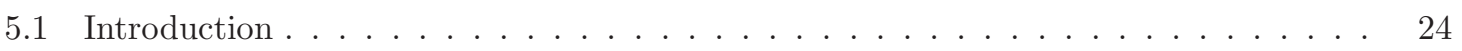

5.2 Time Scaling . . . . . . . . . . . . . . . . . . . . . 24

5.3 Time Scaling Phenomenon in PMU . . . . . . . . . . . . . . . . . . . . . 25

5.4 Experimental Setup . . . . . . . . . . . . . . . . . . . . . . . 28

5.4 .1 Constant frequency . . . . . . . . . . . . . . . . . . . . . . . . . . 29

5.4 .2 Sinusoidally Varying Frequency . . . . . . . . . . . . . . . . . 31

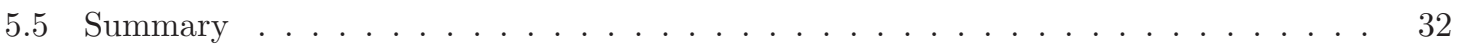

6 Conclusion and Future Work 33

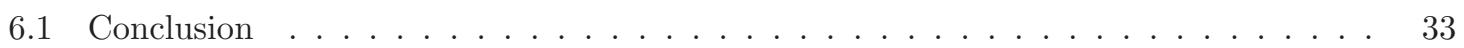

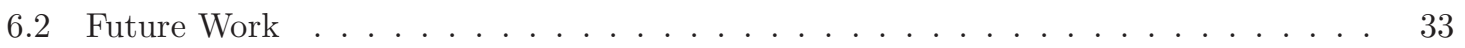




\section{List of Tables}

3.1 Components of Phase A Voltage . . . . . . . . . . . . . . . . . . . . 11

3.2 Amplitude Error . . . . . . . . . . . . . . . . . . . . . . . . . . . . 12

3.3 Phase Angle Error . . . . . . . . . . . . . . . . . . . . . . . . . . . . 12

4.1 Frequency Error $(\mathrm{mHz})$ for Phasors Obtained from DFT . . . . . . . . . . . . . 19

4.2 Frequency Error $(\mathrm{Hz})$ for Phasors Obtained from PLL . . . . . . . . . . . . . . . . 23 


\section{List of Plots}

3.1 PLL based phasor estimator. . . . . . . . . . . . . . . . . . . . 11

3.2 Amplitude Error. . . . . . . . . . . . . . . . . . . . . . . . . . . . 12

3.3 Phase Angle Error. . . . . . . . . . . . . . . . . . . . . . . . . . . . . 12

4.1 Frequency Error in CLS method for DFT based phasor inputs. . . . . . . . . . . . . 20

4.2 Frequency Error in Prony method for DFT based phasor inputs. . . . . . . . . . . . 20

4.3 Frequency Error in PF method for DFT based phasor inputs. . . . . . . . . . . . . . 21

4.4 Frequency Error in CLS method for PLL based phasor inputs. . . . . . . . . . . . . 21

4.5 Frequency Error in Prony method for PLL based phasor inputs. . . . . . . . . . . . . 22

4.6 Frequency Error in PF method for PLL based phasor inputs. . . . . . . . . . . . . . 22

5.1 PMU Block Diagram. . . . . . . . . . . . . . . . . . . . . . . . 26

5.2 Experimental setup using eMEGAsim. . . . . . . . . . . . . . . . . . 28

5.3 Amplitude Plot. . . . . . . . . . . . . . . . . . . . . . . . . . 29

5.4 Phase angle Without Correction. . . . . . . . . . . . . . . . . . . . . . . . . 29

5.5 Phase Angle referred to Target 1 time reference from the start instant of Target 2. . 30

5.6 Phase Angle referred to Target 1 Start Instant. . . . . . . . . . . . . . . . . . . . . 30

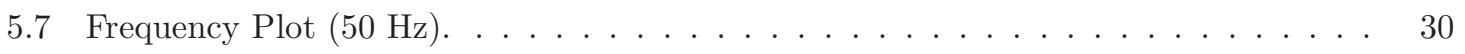

5.8 Phase angle Without Correction. . . . . . . . . . . . . . . . . . . . . . . 31

5.9 Phase Angle referred to Target 1 time reference from the start instant of Target 2. . 31

5.10 Phase Angle referred to Target 1 Start Instant. . . . . . . . . . . . . . . . . . . . . 31

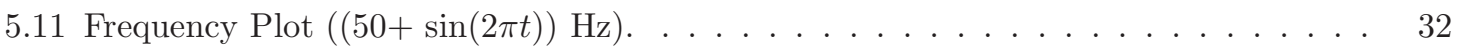




\title{
Nomenclature
}

\author{
Symbols Description \\ $\hat{f} \quad$ Estimated frequency $(\mathrm{Hz})$. \\ $f_{0} \quad$ Nominal frequency $(\mathrm{Hz})$. \\ $F_{s} \quad$ Sampling frequnecy (Samples/s). \\ L Sampling window length. \\ $N \quad$ Sampling per nominal frequency cycle of input \\ analog signal. \\ $r \quad$ Index representing the sampling instant. \\ $\Delta T \quad$ Sampling time interval (s). \\ $\bar{V} \quad$ Actual phasor. \\ $V_{m} \quad$ Actual peak amplitude of the signal. \\ $\omega \quad$ Angular frequency $(\mathrm{rad} / \mathrm{s})$. \\ $\phi \quad$ Actual phase angle of the signal (rad). \\ $\hat{\phi} \quad$ Estimated phase angle for dynamic time ref- \\ erence (rad). \\ $\widetilde{\phi} \quad$ Estimated phase angle for static time reference \\ (rad).
}




\section{Chapter 1}

\section{Introduction}

The electrical power generation, transmission, and distribution networks have become an essential part to our modern society. The electrical power system is complex interconnected system and interconnection among different networks improves the reliability of the system. It is a critical issue for the power system engineers to keep the power grid stable and reliable. The loads that are connected to the power system changes randomly in real-time. Hence, the generation must be changed accordingly to satisfy the demands. Wide scale blackouts have occurred during the past decades due to the unavailability of the system parameters in real-time. Real-time data of power system quantities has many applications ranging from rapid control action like relay functioning to slow function like expansion planning. With high speed real-time measurement, the reliability of power system can maintained by taking proper control actions before the happening of any contingencies.

Supervisory Control and Data Acquisition (SCADA) is an existing real-time monitoring and control system that is available for power systems. Due to high latency in data flow and slower estimation techniques used, the real-time dynamic variations in power system quantities are not observed by SCADA. The system frequency can change over a small range due to generation-load mismatch. The phasor measured describes the present operating state of the system. With the progress in the power industry, the usage of power electronic devices have increased. The nonlinear characteristics of these equipments have introduced harmonic contamination of power system signals. It is therefore, essential for the utilities to seek and depend on reliable models for accurate estimation of the frequency and phasors.

With the power system network expanding day by day, the need for monitoring the interconnected network over a wide area has become an requirement. This led to the development of Wide Area Measurement System (WAMS). angle that are measured in power system at their respective buses are connected. It helps us to observe the power system synchronously with an elaborate time scale. The Phasor Measurement Unit (PMU) and Phasor Data Concentrator (PDC) forms an integral part in WAMS. The voltage phase angles at different buses are closely linked to the behaviour of the network. The phase angles at the buses are measured with respect to local time references. Hence, comparison among those are difficult and that leads to inaccuracy in power flow estimation. With the advent of synchrophasor measurement, which involves in providing common time reference to the estimated phasors, the comparison among the phase angles are made accurate and simpler. 
Synchronized phasor measurement made with PMU provides high speed and coherent real-time information of power system that are not available in the traditional SCADA systems.

A PMU [1] is defined as a device that produces synchronized phasor (synchrophasor) and frequency estimates from voltage/current signals with respect to global time reference. With the development of wide area measurement system (WAMS), the comparison of phasors measured at different location is required. Synchrophasors [2] are defined as the phasors calculated from data samples using a standard time signal as the reference for the measurement. Synchrohasor measurement that are made at different locations can be directly compared with certainty. Time synchronization and time tagging are some of the important terminologies in synchrophasor measurements. The time synchronization is helpful in providing a common time reference for phasor measurement made at different location.

The time stamping [2] is defined as the time of the theoretical phasor that the estimated phasor represents. They are used in comparing data from different sources when reconstructing a power system event or when merging data from multiple source into one data stream. The time reference in PMU is provided by high accuracy clocks synchronized to coordinated universal time (UTC) like global positioning system (GPS). The pulse sent by the GPS provides the time tag and the time reference that are used for phasor measurement.

The standards that are governing the synchrophasor are IEEE 1344, IEEE C37.118, IEEE C37.244, and IEC 61850. Data obtained from different locations are aligned with respect to their time stamping to create a coherent picture about the present state of the power system. PMU is as an integral part of WAMS and the strategic location of the PMUs is necessary to cover the entire grid [3]. The Phasor Data Concentrator (PDC) forms the next level of hierarchy in WAMS. The PDC is generally used to collect data from several PMUs, rejects bad data and alignment of time stamped data obtained to create a coherent record.

Some of the applications of PMU includes:

- post disturbance analysis;

- stability monitoring;

- thermal overload monitoring;

- state estimation;

- real-time control;

- adaptive protection.

With the motivation to find out the best suited algorithms for phasor and frequency calculation for real-time systems even in the presence of disturbances, a few digital estimation algorithms have been considered for the study. The introduction of digital processors have increased the computational speed significantly. Now, there is a need for digital algorithms which can estimate these system parameters accurately. Some of the available methods in literature include wavelet transform [4], zero crossing [5], modified zero crossing, and signal demodulation [6]. In pursuit of higher accuracy for frequency and phasor measurement, the optimization methods such as least mean square methods [7], Kalman filter [8], and Newton's method [9] are being used. These above optimization methods are not widely used in real time application as convergence being the main problem. In 
addition to the above mentioned algorithms, some of the other phasor measurement techniques that are available in literature include measuring phasors with Discrete Fourier Transform and Taylor series [10], use of artificial neural network [11], S-transform technique [12], Fourier algorithm and novel complex filter [13], and matrix pencil method [14]. The algorithms employed in this thesis for frequency estimation include Prony method [15], Polynomial Fitting (PF) [6], Complex Valued Least Squares (CLS) [16], and Phase Locked Loop (PLL). For phasor estimation, the algorithms employed in this paper are Discrete Fourier Transform (DFT) [17], Phase Locked Loop (PLL), and Smart Discrete Fourier Transform (SDFT) [18].

Time scaling refers to time elapsed between two clocks while measuring the same event. Because of the time scaling phenomenon, the time measured by one clock can either be a scaled up or scaled down value of the time measured by another clock. In real-time phasor measurement, the phasors are measured with respect to the local time reference and are referred back to the global time reference provided by the GPS clock. In this case, the time scaling phenomenon particularly affects the phase angle of the measured phasor. The problem in phase angle measurement mentioned earlier is investigated and a possible solution is suggested to overcome the problem.

\section{Organization of Thesis}

Chapter 2: This chapter covers the literature survey on different frequency and phasor estimation techniques that are available for power systems.

Chapter 3: In this chapter, the concepts of phasor and time synchrnoization are discussed. The different phasor measurement techniques are also explained and compared with each other. A case study is performed using these techniques and the simulation results are presented.

Chapter 4: This chapter covers the different frequency estimation algorithms used in power systems. A detailed case study is performed to analyse the effectiveness of these estimation algorithms. Comparison amongst the different methods is carried out.

Chapter 5: In this chapter, the concept of time scaling is explained in this chapter. The effect of time scaling on phasor measurement is discussed. An experimental setup is built using eMEGAsim simulator to verify the time scaling effect on estimated phasor and frequency quantities.

Chapter 6: This chapter concludes the thesis and discusses the possible future scope of work. 


\section{Chapter 2}

\section{Literature Overview}

The chapter covers a brief literature survey about the various phasor and frequency estimation algorithms that are available for power systems.

\subsection{Phasor Estimation Techniques}

\subsubsection{Newton type algorithm}

V. V. Terzija et al. [9] proposed the Newton's iterative algorithm for phasor and frequency estimation in power system. The estimation problem is considered as an unconstrained optimization problem. An observation model is considered based on the phase voltage signal with DC component and random noise present. Then, the parameter vector consisting of DC offset, amplitude, phase angle, and system frequency is deduced from the model. The measurement vector is formed and jacobian matrix is obtained. The system parameters vector elements are obtained using pseudo inverse technique. The algorithm provides good accuracy even for wide frequency variation and distorted signal containing DC component and random noise. The convergence speed of the algorithm is the main issue here.

\subsubsection{Recursive Gauss-Newton adaptive filter}

A recursive Gauss-Newton algorithm based adaptive filter was proposed by P. K. Das et al. [19] to estimate the fundamental and harmonic components power system phasor and frequency quantities. The adaptive filter coefficients are obtained from the unconstrained optimization of an error function weighted by a variable forgetting factor which is used for quick convergence. The forgetting factor is tuned iteratively and the Gauss-Newton algorithm is used for reducing computational complexity. The algorithm proves to be superior to Newton type algorithm and has good accuracy even if the power system signals contains decaying DC offset and noise components. 


\subsection{Frequency Estimation Techniques}

\subsubsection{Zero-Crossing Methods}

This method is widely used for its simplicity in estimating the system frequency. In the zero crossing approach [6], the time elapsed between two successive zero crossings of the signal is measured to determine the frequency. The accuracy of this method is influenced by zero-crossing detection, harmonics, switching transients, and noise. The tracking ability for this method is slow because a zero-crossing can only be detected after at least half a cycle and it takes more cycles for stable results. By using a supplementary technique, such as polynomial interpolation, the accuracy can be improved. However, the tracking speed is still an issue.

\subsubsection{Discrete Fourier Transform}

The conventional DFT method is able to estimate the system frequency when the system is operating at its nominal frequency. When the the system frequency deviates from its nominal value, error occurs in DFT method [20]. The leakage effect of DFT could bring significant error when the sampling frequency is not an integral multiple of fundamental frequency. Many techniques were proposed such adaptive sampling frequency [21] and adaptive sampling window [22] to deal with this problem. The DFT method is also susceptible to harmonics and noise.

\subsubsection{Extended Complex Kalman filter}

The extended kalman filter model for frequency estimation was proposed by P. K. Dash et al. [8] for estimating the power system frequency. The $\alpha \beta$ transform is used to convert the discrete value of three phase signals into complex voltage vector. The non-linear state space model formulated from the complex voltage vector is used by extended kalman filter approach to iteratively obtain the true state of the model. The convergence speed is increased by considering the harmonics in sate space formulation. For any rise or decay in system frequency this method has been found to work well.

\subsubsection{Least Mean Square}

R. Chudamani et al. [7] proposed the complex form of least mean square to obtain the power system frequency. The discrete value of three phase signals are transformed into complex voltage vector form using $\alpha \beta$ transform. Variable step-size is used to minimize the square of successive error samples and to increase the convergence rate. The advantage of this method is its simplicity in formulation and computational efficiency.

\subsubsection{Nonlinear Least Square}

A non-linear least square technique was proposed by R.Chudamani et al. [23] for estimation of power system frequency. The voltage signals that are at the considered at the measuring point is modelled based on fourier series. The non-linear least square problem of frequency estimation is carried out over a range of allowable frequency variation and since, only one parameter (i.e, frequency) is enough to minimize the error an 1-D search is used. The algorithm is also capable of finding the harmonics present in the system. 


\section{Chapter 3}

\section{Phasor Measurement Techniques}

The concept of using phasor to describe the power system quantities dates back to 19th century and Charles P. Steinmetz was the originator of the phasor transform. Phasors are mainly used for analyzing alternating voltage and current quantities. The phasors estimated in power systems are used in applications such as wide area protection and emergency control, and state estimation. In the next section a brief description about phasor is presented.

\subsection{Concept of Phasor}

A phasor can be defined as a complex equivalent of a sinusoidal quantity whose amplitude, angular frequency and phase angle are time invariant. The phasor defined above is known as static phasor. Consider a sinusoidal input signal of frequency $\omega=2 \pi f_{0}$ as follows,

$$
\begin{aligned}
& v(t)=V_{m} \cos (\theta(t)) \\
& \theta(t)=\omega t+\phi .
\end{aligned}
$$

Its phasor representation is given by,

$$
\bar{V}=\frac{V_{m}}{\sqrt{2}} \angle \phi .
$$

Phasor representation is possible only for single frequency component in a sinusoid. In real time systems, a sinusoid is often corrupted by harmonics and noise. So, it is necessary to extract the single frequency component of the signal (usually the fundamental one) and then represent it by a phasor.

The phase angle $(\phi)$ in a phasor is measured with respect to a specific time variable. The time variable is specified with respect to a reference time instant. The time reference can be defined as instant at which the specified time variable is set to zero. The phase angle given in (3.3) is measured with respect to the time reference $t=0$. Now, consider that the time reference is shifted to $t=T$. When the time reference is shifted, the time variable also changes. The time variable is now changed to $\tau$. The relation between $t$ and $\tau$ is given by $t=T+\tau$. Now, representing (3.2) with respect to 
the new time variable $\tau$, we get,

$$
\begin{aligned}
& v(\tau)=V_{m} \cos (\omega(\tau+T)+\phi) \\
& v(\tau)=V_{m} \cos (\omega \tau+\omega T+\phi) .
\end{aligned}
$$

The phasor representation of (3.2) with respect to reference time instant $\tau=0$ (or $t=T$ ) is given by,

$$
\bar{V}_{n e w}=\frac{V_{m}}{\sqrt{2}} \angle\{\phi+\omega T\} .
$$

Time synchronization is required for providing a common time reference for phasor measurement made at different locations. The relationship between the measured phasor and the time reference is such that the phase angle of the phasor is equal to the angular separation between the measuring instant and the peak of the sinusoid. In power system, the active and reactive power flow in the grid are calculated using the power flow equations based on the phasor values of the corresponding voltage and current quantities. The voltage and current quantities that are considered are expressed with respect to the same time variable. This in turn, indicates that the time reference should be considered to obtain all the voltage and current phasors. The relative phase angle between the voltage and current phasors can be directly obtained as they are measured with respect to the same time reference. Time synchronization in PMU is provided by the GPS pulse signal.

The phasor concept was initially developed for pure sinusoidal signals. However, the concept can be extended in the form of dynamic phasor for signals whose amplitude and phase angle are slowly varying with time. The techniques that are available for estimating static phasors may also be suitable for dynamic phasors. The different methods for calculating the fundamental phasors are discussed in the following sections.

\subsection{Phasor Estimation Techniques}

\subsubsection{Discrete Fourier Transform}

DFT is a technique used in signal processing to calculate the amplitude and phase angle of individual signal frequency components. The sinusoidal signal given in (3.2) is sampled at $N$ times per cycle such that $F_{s}=N f_{0}$. The sampled domain representation of signal is given by,

$$
v[n]=V_{m} \cos (\omega n \Delta T+\phi) .
$$

For real-time estimation of amplitude and angle, the phasors that are obtained must be regularly updated. There are two ways for updating phasor. The estimated phasors can be represented with respect to two time references namely,

- dynamic time reference $(t=r \Delta T)$

- static time reference $(t=0)$ 


\section{Dynamic Time Reference}

The phasor of the $k$ th harmonic component of $v[n]$ is shown below,

$$
\begin{aligned}
\hat{\bar{V}}_{k}[r] & =\frac{2}{N} \sum_{n=M}^{N-1+M} v[r+n] e^{-j k n \varphi} \\
\varphi & =\frac{2 \pi}{N} .
\end{aligned}
$$

The value of $M$ can be set to zero, if the phasors are calculated based on the future samples. But, this introduces a time delay of of one cycle in phasor estimation. The value of $M$ is set to $1-N$, when the phasors are calculated based on the past signal samples. Equation (3.8), represents the non-recursive representation of $v[n]$. The recursive form representation is represented below,

$$
\hat{\bar{V}}_{k}[r+1]=\left(\hat{\bar{V}}_{k}[r]+\frac{2}{N}\{v[r+N+M]-v[r+M]\} e^{-j M k \varphi}\right) e^{j k \varphi} .
$$

It is clear from the above that the resulting phasor of a constant sinusoid with nominal power system frequency obtained from the above method will have constant magnitude and phase anngle will rotate in counterclockwise direction by an angle $\varphi$ as the data advances by one sample.

\section{Static Time reference}

The phasor of the $k$ th harmonic component of $v[n]$ based on static time reference shown below,

$$
\widetilde{\bar{V}}_{k}[r]=\frac{2}{N} \sum_{n=M}^{N-1+M} v[r+n] e^{-j \varphi k(n+r)}
$$

The recursive form of the above equation is represented below,

$$
\widetilde{\bar{V}}_{k}[r+1]=\widetilde{\bar{V}}_{k}[r]+\frac{2}{N}\{v[r+N+M]-v[r+M]\} e^{-j(M+r) k \varphi} .
$$

Equations (3.8) and (3.11), represents the non-recursive formulation of phasor estimation. Since, the phasor calculations are performed fresh for each window without using any data from earlier estimates, this algorithm is known as "Non-recursive algorithm". From (3.10) and (3.12), it can be observed that the new phasor is calculated by making a recursive update on the old phasor. Hence, this algorithm is known as "Recursive algorithm". If the input signal is assumed to be a pure sinusoid, the second term in (3.10) and (3.12) gets cancelled and only the first term in (3.10) and (3.12) prevails.

\subsubsection{Smart Discrete Fourier Transform}

The SDFT algorithm is similar to the conventional DFT method. The SDFT method was originally proposed for a constant frequency signal. However, unlike the ideal case for ordinary DFT, the signal frequency can be different from the nominal frequency. The phasor estimation in SDFT method involves in first calculating the system frequency and based on the deviation of the frequency from its nominal value the phasors are calculated. The phasors that are obtained from DFT and are used for frequency estimation forms the raw phasors. The phasors that are calculated based frequency 
deviation forms the updated phasors. The equation given in (3.7) can also be represented as,

$$
v[n]=\frac{\bar{V} e^{j(\omega n \Delta T)}+\bar{V}^{*} e^{-j(\omega n \Delta T)}}{2} .
$$

Combining the fundamental phasor (i.e, $k=1$ ) obtained from (3.8) and (3.13) and taking frequency deviation $\left(\omega=2 \pi\left(f_{0}+\Delta f\right)\right)$ into consideration, we obtain,

$$
\hat{\bar{V}}_{1}(r)=a[r]+b[r] .
$$

The terms $a[r], b[r]$, and $c$ are shown below,

$$
\begin{gathered}
a[r]=\frac{\bar{V}}{N} \frac{\sin \left(\frac{\pi \Delta f}{f_{0}}\right)}{\sin \left(\frac{\pi \Delta f}{N f_{0}}\right)} e^{j \frac{\pi}{N f_{0}}\left(\Delta f(2 r+N-1)+2 f_{0} r\right)} \\
b[r]=\frac{\bar{V}^{*}}{N} \frac{\sin \left(\frac{\pi\left(2 f_{0}+\Delta f\right)}{f_{0}}\right)}{\sin \left(\frac{\pi\left(2 f_{0}+\Delta f\right)}{N f_{0}}\right)} e^{\frac{-j \pi}{N f_{0}}\left(r \Delta f+\left(2 f_{0}+\Delta f\right)(r+N-1)\right)} \\
c=e^{j \frac{2 \pi\left(f_{0}+\Delta f\right)}{N f_{0}}} .
\end{gathered}
$$

Once the frequency deviation is estimated, the phasor is calculated from (3.15) using the following relation,

$$
\bar{V}=a[r] \frac{N \sin \left(\frac{\pi \Delta f}{N f_{0}}\right)}{\sin \left(\frac{\pi \Delta f}{f_{0}}\right)} e^{-j \frac{\pi}{N f_{0}}\left(\Delta f(2 r+N-1)+2 f_{0} r\right)} .
$$

SDFT keeps both the advantage of normal DFT and also deals with the difficulty of frequency deviation errors while taking harmonics and noise into consideration. SDFT is found to be more accurate and harmonic resisting method.

\subsubsection{Phase Locked Loop}

The PLL technique has been used as a common way of recovering the phase angle and frequency information in electrical systems. The schematic diagram of PLL based phasor estimator is shown in Fig. 3.1.

The input signals which are in abc domain are first converted to dq domain using a transformation matrix $T$. The transformation matrix considered here is given below,

$$
T=\sqrt{\frac{2}{3}}\left[\begin{array}{ccc}
\cos (\theta) & \cos \left(\theta-\frac{2 \pi}{3}\right) & \cos \left(\theta+\frac{2 \pi}{3}\right) \\
-\sin (\theta) & -\sin \left(\theta-\frac{2 \pi}{3}\right) & -\sin \left(\theta+\frac{2 \pi}{3}\right)
\end{array}\right] .
$$

A first order PI controller is used to obtain the frequency information of the input signals. When the input signals are unbalanced, there is the presence of double frequency component in $V_{d}$ and $V_{q}$. A low pass filter (LPF) is used to filter out these high-frequency components. The imaginary component of the dq phasor is passed through the PI controller to obtain the value of $\theta$. The 


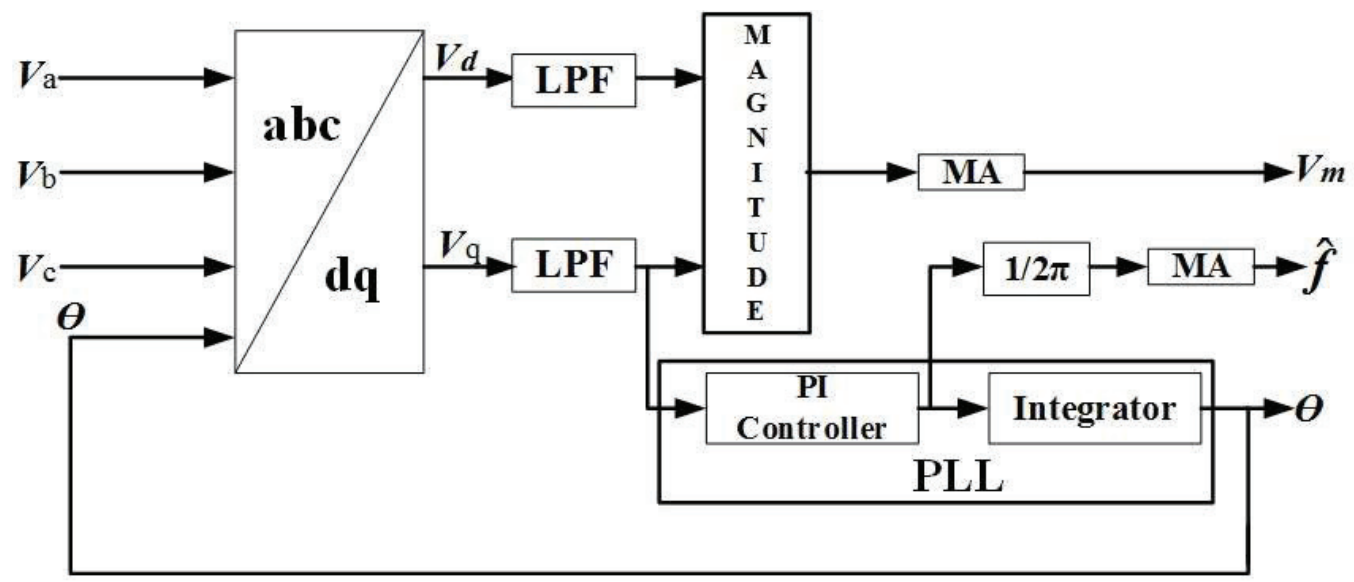

Figure 3.1: PLL based phasor estimator.

frequency information of the input signal is obtained by passing the $\theta$ through an integrator. There is a requirement of automatic gain controller if the input signal parameters are varying over a wide range. The moving average block (MA) is used to smoothen the magnitude and frequency waveform by reducing the ripples present in it.

\subsection{Simulation Results}

A case study is performed on a three-phase voltage signal that contains both the fundamental frequency and harmonic components. Only the third and fifth harmonic components are considered for the signal. There are all sequence (i.e., positive, negative, and zero) components at the fundamental frequency. However, no negative or zero sequence components at harmonic frequency. The amplitude of all fundamental frequency components is taken to be time varying. Table 3.1, shows the amplitude and initial phase angles (corresponding to phase A) of different components of the test voltage signal. The simulations are carried out in MATLAB/Simulink. The sampling frequency is set at $12.8 \mathrm{kHz}$. The nominal system frequency is considered to be $50 \mathrm{~Hz}$ and the number of samples per cycle is 256 (i.e., $N=256$ ). Harmonic components are added to these signals to verify the effectiveness of the phasor estimation algorithms.

Table 3.1: Components of Phase A Voltage

\begin{tabular}{|c|c|c|}
\hline Components & amplitude (V) & phase angle (rad) \\
\hline Positive sequence & $100+10 \sin (2 \pi t)$ & 0 \\
\hline Negative sequence & $50+5 \sin (2 \pi t)$ & -1.0491 \\
\hline Zero sequence & $30+3 \sin (2 \pi t)$ & -2.0769 \\
\hline Third harmonic & 12 & 0 \\
\hline Fifth harmonic & 9.5 & 0 \\
\hline
\end{tabular}

The system frequency is assumed to be sinusoidally varying with a time period of one second between $49 \mathrm{~Hz}$ and $51 \mathrm{~Hz}$. The amplitude errors (absolute value) and the phase angle errors (absolute value) for the different phasor estimation algorithm are tabulated in Table 3.2. The amplitude error is minimum in the case of SDFT method. The amplitude error are produced in Fig 3.2. From Table 
Table 3.2: Amplitude Error

\begin{tabular}{|c|c|c|c|}
\hline Methods & DFT & PLL & SDFT \\
\hline Amplitude error (V) & 0.09 & 0.244 & 0.04 \\
\hline
\end{tabular}

Table 3.3: Phase Angle Error

\begin{tabular}{|c|c|c|c|}
\hline Methods & DFT & PLL & SDFT \\
\hline Phase angle error (rad) & $7.3 \mathrm{e}-3$ & 0.03 & $3.6 \mathrm{e}-3$ \\
\hline
\end{tabular}

3.3, it is found that the phase angle error is also minimum in the case of SDFT method. The plots of phase angle error are shown in Fig 3.3.

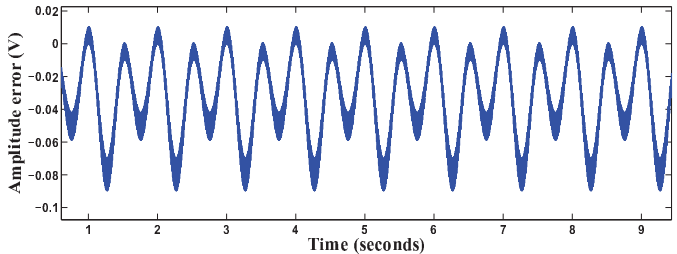

(a) DFT

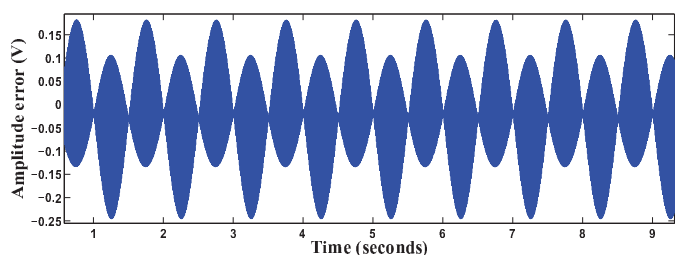

(b) PLL

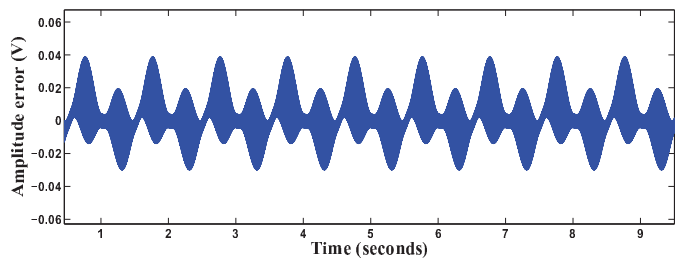

(c) SDFT

Figure 3.2: Amplitude Error.

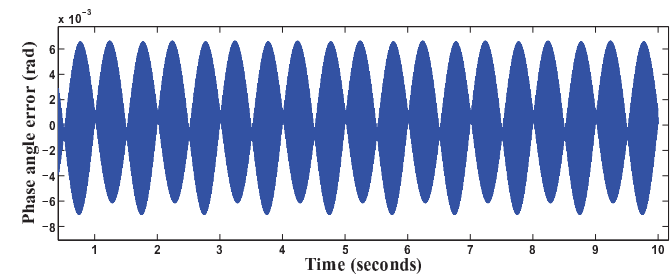

(a) DFT

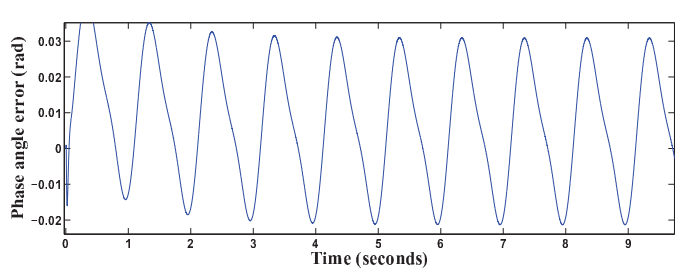

(b) PLL

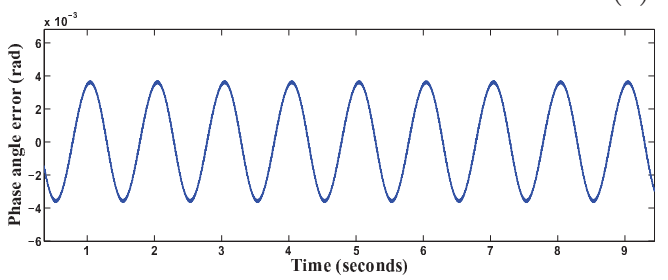

(c) SDFT

Figure 3.3: Phase Angle Error. 


\subsection{Summary}

In this chapter, an overview about the concept of phasor and time synchronization are provided. The different phasor measurement techniques are explained and the comparison among the techniques is presented with a case study. Finally, it is observed that the SDFT technique provides accurate amplitude and phase angle measurement since the phasors are calculated based on the frequency deviation from its nominal value. 


\section{Chapter 4}

\section{Frequency Measurement Techniques}

The power system signals are often contaminated with harmonics. So, the signal fundamental frequency component must be estimated for calculating the system frequency. The operating system frequency is estimated from the positive sequence phasor at fundamental frequency. The fundamental phasor obtained either from DFT or PLL are used as inputs for the frequency estimation algorithms. The estimation algorithms must be robust in nature in order to provide accurate output under dynamic operating condition such frequency variation, harmonic contamination, DC offset, and noise. The computational time required is also considered as an important requirement for these estimation algorithms. The different frequency estimation algorithms are explained in the following sections.

\subsection{Complex Valued Least Square}

The original CLS method given by [18] is based on scalar model (i.e., $L=1$ ). From (3.15), (3.16), and (3.17) we get,

$$
\begin{gathered}
a[r]=c a[r-1] \\
b[r]=c^{-1} b[r-1] .
\end{gathered}
$$

Then we can write,

$$
\begin{aligned}
\hat{\bar{V}}_{1}[r] & =c a[r-1]+c^{-1} b[r-1] \\
\hat{\bar{V}}_{1}[r-1] & =a[r-1]+b[r-1] \\
\hat{\bar{V}}_{1}[r-2] & =a[r-2]+b[r-2] \\
& =c^{-1} a[r-1]+c b[r-1] .
\end{aligned}
$$


After some algebraic manipulations, the following relationship is obtained,

$$
\begin{aligned}
w \hat{\bar{V}}_{1}[r-1] & =\hat{\bar{V}}_{1}[r]+\hat{\bar{V}}_{1}[r-2] \\
w & =c+c^{-1} .
\end{aligned}
$$

In the vector model [16], a sliding window of $L$ consecutive phasor samples are considered. Equation (4.6) can be written in vector form over a window of $\mathrm{L}$ samples as follows,

$$
\left[\begin{array}{c}
\hat{\bar{V}}_{1}[r-1] \\
\hat{\bar{V}}_{1}[r-2] \\
\cdot \\
\cdot \\
\hat{\bar{V}}_{1}[r-L]
\end{array}\right] w=\left[\begin{array}{c}
\hat{\bar{V}}_{1}[r] \\
\hat{\bar{V}}_{1}[r-1] \\
\cdot \\
\cdot \\
\hat{\bar{V}}_{1}[r-L+1]
\end{array}\right]+\left[\begin{array}{c}
\hat{\bar{V}}_{1}[r-2] \\
\hat{\bar{V}}_{1}[r-3] \\
\cdot \\
\cdot \\
\hat{\bar{V}}_{1}[r-L-1]
\end{array}\right] .
$$

We define here,

$$
\mathbf{V}_{1}[r]=\left[\hat{\bar{V}}_{1}[r] \ldots \hat{\bar{V}}_{1}[r-L+1]\right]^{T}
$$

Which gives,

$$
\mathbf{V}_{1}[r-1] w=\mathbf{V}_{1}[r]+\mathbf{V}_{1}[r-2]
$$

Here, $w$ is real valued parameter. The error vector is of the form,

$$
e[r]=\mathbf{V}_{1}[r-1] w-\left\{\mathbf{V}_{1}[r]+\mathbf{V}_{1}[r-2]\right\} .
$$

Its Hermitian transpose is given by,

$$
e^{H}[r]=\mathbf{V}_{1}^{H}[r-1] w-\left\{\mathbf{V}_{1}^{H}[r]+\mathbf{V}_{1}^{H}[r-2]\right\} .
$$

The optimal value of $w$ is calculated using complex valued least squares. It aims to find the optimal value of $w$ to minimize the total mean square error over a number of available observations.

$$
\begin{gathered}
J(w)=e^{H}[r] e[r] \\
=\left\|\mathbf{V}_{1}[r-1]\right\|^{2} w^{2}+\left\|\mathbf{V}_{1}[r]+\mathbf{V}_{1}[r-2]\right\|^{2}-2 \operatorname{Re}\left(\mathbf{V}_{1}^{H}[r-1]\left\{\mathbf{V}_{1}[r]+\mathbf{V}_{1}[r-2]\right\}\right) w .
\end{gathered}
$$

The partial derivative $\frac{\partial J(w)}{\partial w}$ becomes,

$$
\frac{\partial J(w)}{\partial w}=2\left\|\mathbf{V}_{1}[r-1]\right\|^{2} w-2 \operatorname{Re}\left(\mathbf{V}_{1}^{H}[r-1]\left\{\mathbf{V}_{1}[r]+\widetilde{V}_{1}[r-2]\right\}\right) .
$$


Then the partial derivative $\frac{\partial J(w)}{\partial w}$ is set to zero to obtain the solution of $w$. Finally, the expression of $w$ is represented by,

$$
w=\frac{\operatorname{Re}\left(\mathbf{V}_{1}[r-1]^{H}\left\{\mathbf{V}_{1}[r]+\mathbf{V}_{1}[r-2]\right\}\right)}{\left\|\mathbf{V}_{1}[r-1]\right\|^{2}}
$$

Once the value of $w$ is calculated the system frequency is estimated as shown below,

$$
\hat{f}[r-L-1]=\frac{F_{s}}{2 \pi} \cos ^{-1}\left(\frac{w}{2}\right) .
$$

\subsection{Prony Method}

The sampled input waveform is first filtered and then the positive sequence phasor is calculated. Only the real part of the phasor is required for the frequency estimation. When the input signal frequency changes the inherent DFT rectangular window has some disadvantages. To improve the filtering smoothing window such as Hamming window or Blackman window are used. The prefiltering is done to improve the accuracy of frequency estimation. The filtered real part of the phasor can be approximated into one sinusoid given,

$$
Q[r]=A \cos (r \omega \Delta T+\psi) .
$$

In complex form, (4.18) can be written as,

$$
Q[r]=b z_{1}^{r}+b^{*} z_{1}^{* r}
$$

The terms $Y$ and $z_{1}$ are represented below,

$$
\begin{aligned}
& z_{1}=e^{j \omega T} \\
& b=\frac{A}{2} e^{j \psi} .
\end{aligned}
$$

The estimation problem is to find $b$ and $z_{1}$ so that the error,

$$
\delta[r]=\operatorname{Re}\left\{\hat{\bar{V}}_{1}[r]\right\}-Q[r] .
$$

will be minimized. The Prony's estimation method converts this nonlinear problem into a linear fitting problem by minimizing the error $E$ defined by,

$$
E=\sum_{r=p}^{L-1}(\epsilon[r])^{2}
$$

Where $p$ is the number of exponents and $\epsilon[r]$ is defined as,

$$
\epsilon[r]=\sum_{h=0}^{p} x_{h} \delta[h+r-1] .
$$


The parameter $x_{h}$ is initially unknown, and are related to the frequency of the sinusoid. The key step to the estimation is to recognize that the (4.19) is solution to some linear constant coefficient difference equation. In order to find the form of the difference equation, the polynomial $H(z)$ is defined for $\mathrm{p}=2$.

$$
H(z)=x_{0}\left(z-z_{1}\right)\left(z-z_{1}^{*}\right)=0
$$

The exponents $z_{1}$ and $z_{1}^{*}$ are the roots of the polynomial. Using (4.19) we obtain,

$$
\sum_{h=0}^{2} x_{h} Q[h+r-1]=x_{0} Q[r-1]+x_{1} Q[r]+x_{2} Q[r+1]=0 .
$$

From (4.22) and (4.24) it follows that

$$
\begin{aligned}
\epsilon[r] & =\sum_{h=0}^{2} x_{h}\left(\operatorname{Re}\left\{\hat{\bar{V}}_{1}[h+r-1]\right\}-Q[h+r-1]\right), \\
& =x_{0} \operatorname{Re}\left\{\hat{\bar{V}}_{1}[r-1]\right\}+x_{1} \operatorname{Re}\left\{\hat{\bar{V}}_{1}[r]\right\}+x_{2} \operatorname{Re}\left\{\hat{\bar{V}}_{1}[r+1]\right\} .
\end{aligned}
$$

If $z_{1}$ is a root of the polynomial $H(z)$, then $z_{1}^{-1}$ is also a root. So, the coefficients are symmetric about $x_{1}$ (i.e., $x_{0}=x_{2}$ ). It is convenient to chose $x_{0}$ so that $x_{1}=1$. For $x_{0}=x_{2}, x_{1}=1$

$$
\epsilon[r]=\operatorname{Re}\left\{\hat{\bar{V}}_{1}[r]\right\}+x_{0}\left(\operatorname{Re}\left\{\hat{\bar{V}}_{1}[r-1]\right\}+\operatorname{Re}\left\{\hat{\bar{V}}_{1}[r+1]\right\}\right) .
$$

The minimization of $E$ with respect to the unknown $x_{0}$ will be achieved by,

$$
\frac{\partial E}{\partial x_{0}}=\sum_{r=2}^{L-1} 2\left(\operatorname{Re}\left\{\hat{\bar{V}}_{1}[r]\right\}+x_{0} \operatorname{Re}\left\{\hat{\bar{V}}_{1}[r-1]\right\}+\operatorname{Re}\left\{\hat{\bar{V}}_{1}[r+1]\right\}\right)\left(\operatorname{Re}\left\{\hat{\bar{V}}_{1}[r-1]\right\}+\operatorname{Re}\left\{\hat{\bar{V}}_{1}[r+1]\right\}\right) .(
$$

Upon setting the partial derivative to zero. The solution to $x_{0}$ from (4.30) is given by,

$$
x_{0}=-\frac{\sum_{r=2}^{L-1} \operatorname{Re}\left\{\hat{\bar{V}}_{1}[r]\right\}\left(\operatorname{Re}\left\{\hat{\bar{V}}_{1}[r-1]+\hat{\bar{V}}_{1}[r+1]\right\}\right)}{\sum_{r=2}^{L-1}\left(\operatorname{Re}\left\{\hat{\bar{V}}_{1}[r-1]\right\}+\operatorname{Re}\left\{\hat{\bar{V}}_{1}[r+1]\right\}\right)^{2}} .
$$

The polynomial $H(z)$ can be expressed as,

$$
z^{2}+\frac{1}{x_{0}} z+1=0
$$

The roots of the polynomial are,

$$
z=-\frac{1}{2 x_{0}} \pm j \sqrt{1-\frac{1}{4 x_{0}^{2}}}
$$


Since the roots are defined as (4.19)

$$
\begin{aligned}
z & =e^{ \pm j \omega T}, \\
& =\cos (\omega T) \pm j \sin (\omega T) .
\end{aligned}
$$

Comparing (4.33) and (4.35), the system frequency is given by,

$$
\hat{f}[r-1]=\frac{F_{s}}{2 \pi} \cos ^{-1}\left(\frac{1}{2 x_{0}}\right) .
$$

\subsection{Polynomial Fitting}

A sliding data window of size $L$ which contains the phase angles of the positive sequence phasor is constructed. It is represented by,

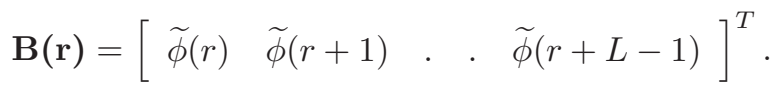

Let us consider a $l$ th degree for $\phi(t)$ given by,

$$
\phi(t)=g_{0}+g_{1} t+\ldots+g_{l} t^{l} .
$$

The polynomial is fitted using the least square technique [6].

$$
R G=\mathbf{B}[\mathbf{r}]
$$

The terms $R$ and $G$ are shown below,

$$
\begin{aligned}
& \mathbf{R}=\left[\begin{array}{cccc}
1 & (r+1) \Delta T & . . & (r+1)^{l}(\Delta T)^{l} \\
1 & (r+2) \Delta T & . . & (r+2)^{l}(\Delta T)^{l} \\
1 & (r+3) \Delta T & . . & (r+3)^{l}(\Delta T)^{l} \\
\cdot & \cdot & . . & . \\
1 & (r+L-1) \Delta T & . . & (r+L-1)^{l}(\Delta T)^{l}
\end{array}\right] \\
& \mathbf{G}=\left[\begin{array}{lllll}
g_{0} & g_{1} & . & \cdot & g_{l}
\end{array}\right]^{T} .
\end{aligned}
$$

The coefficients of the polynomial in (4.38) are calculated from (4.39) using the pseudo-inverse technique given by,

$$
\mathbf{D}=\left(\mathbf{R}^{T} \mathbf{R}\right)^{-1} \mathbf{R}^{T} \mathbf{B}[\mathbf{r}] .
$$

The instantaneous frequency $f(t)$ is calculated using the following relationship,

$$
\begin{aligned}
f(t) & =\frac{1}{2 \pi} \frac{d \theta(t)}{d t}, \\
& =f_{0}+\frac{1}{2 \pi}\left(g_{1}+2 g_{2} t+\ldots+l g_{l} t^{l-1}\right) .
\end{aligned}
$$


The continuous time frequency $f(t)$ is discretized. The frequency is evaluated at the end of the data window to get the recent estimate of system frequency represented by,

$$
\hat{f}[r+L-1]=f_{0}+\frac{1}{2 \pi} \sum_{y=0}^{l-1} y g_{y}\{\Delta T(r+L-1)\}^{y-1} .
$$

The attractive simplicity of this method makes it very easy for real time implementation. The noise and harmonic suppression are also good in this method. The instantaneous frequency prediction is possible within a reasonable range, by extrapolating the polynomial outside the data window.

\subsection{Simulation Results}

The case study is performed with a three-phase voltage signal that contains both the fundamental and harmonic components. The signal components corresponding to phase A voltage is tabulated in Table 3.2. The simulation are carried out in MATLAB/Simulink and the sampling frequency is set at $12.8 \mathrm{kHz}$. The case study is presented in the following section.

\section{Sinusoidally varying frequency}

Consider that the system frequency is a slow varying sinusoid given by,

$$
f=50+\sin (2 \pi t)
$$

Therefore, the signal frequency oscillates between $49 \mathrm{~Hz}$ and $51 \mathrm{~Hz}$ with a time period of one second. The number of phasor samples required for frequency estimation algorithms is varied from quarter cycle to three cycle window length. The output frequency waveforms are passed through moving average filter to reduce the ripples present in it.

The frequency error (absolute value)for different estimation algorithm whose phasors are obtained from DFT are tabulated in Tabel 4.1.

Table 4.1: Frequency Error $(\mathrm{mHz})$ for Phasors Obtained from DFT

\begin{tabular}{|c|c|c|c|c|c|}
\hline \multirow{2}{*}{ Methods } & \multicolumn{5}{|c|}{ Window length } \\
\cline { 2 - 6 } & QC & HC & OC & TC & THC \\
\hline CLS & 16.5 & 1.95 & 2.4 & 4.4 & 7.57 \\
\hline Prony & 40 & 4.15 & 4.49 & 6 & 9.1 \\
\hline PF & 20 & 15 & 3.1 & 5.9 & 13.2 \\
\hline HC-half cycle, OC-one cycle, QC-quarter cycle \\
TC-two cycles, THC-Three cycles \\
\hline
\end{tabular}

From Table 4.1, it can be seen that the frequency error is least for CLS method compared to the other frequency estimation algorithms. The half cycle window length is suggested for CLS and prony method for obtaining the minimum frequency error. When the polynomial fitting method is used for frequency estimation, one cycle window length is used for obtaining minimum frequency error. The errors in frequency estimation by different algorithms with DFT-based phasor input are plotted in Fig. 4.1, 4.2, and 4.3. 


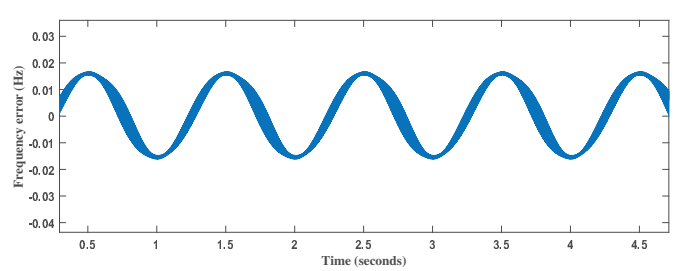

(a) Quarter cycle

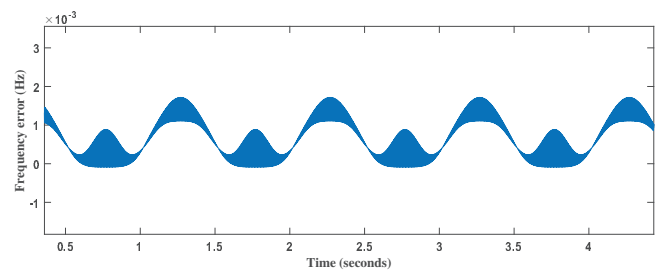

(b) Half cycle

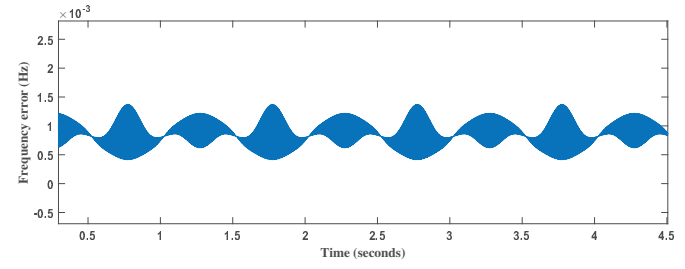

(c) One cycle

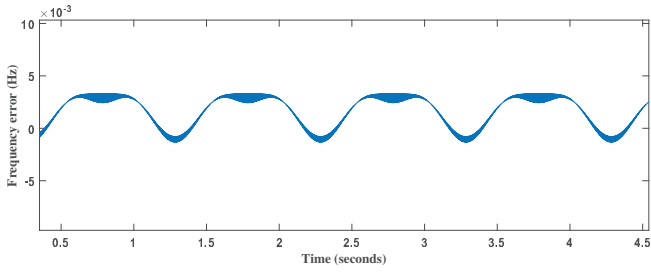

(d) Two cycles

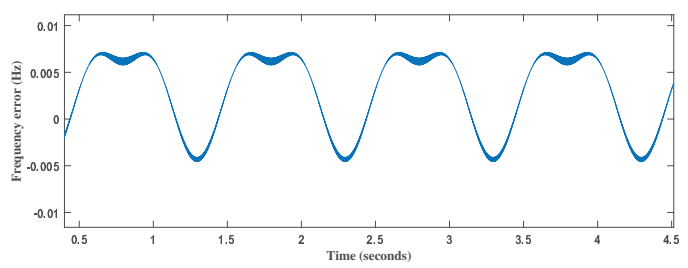

(e) Three cycles

Figure 4.1: Frequency Error in CLS method for DFT based phasor inputs.

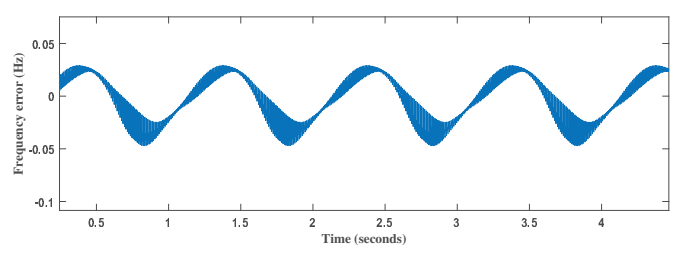

(a) Quarter cycle

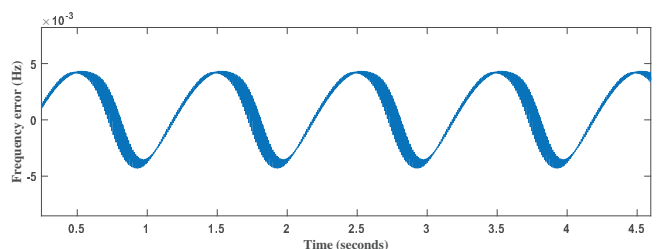

(b) Half cycle

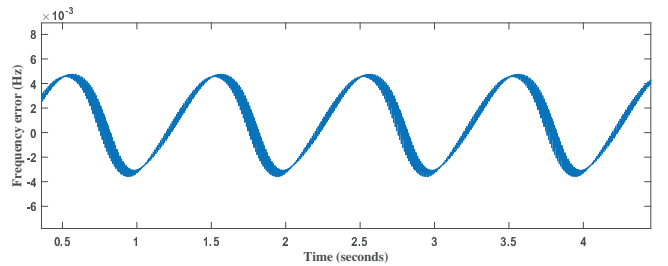

(c) One cycle

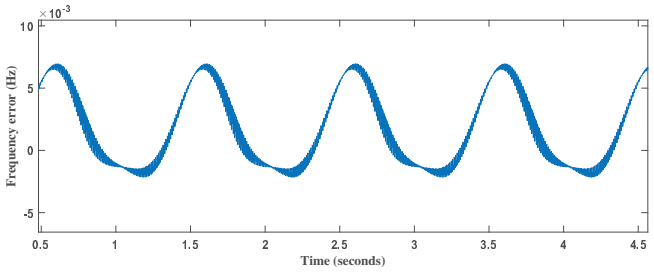

(d) Two cycles

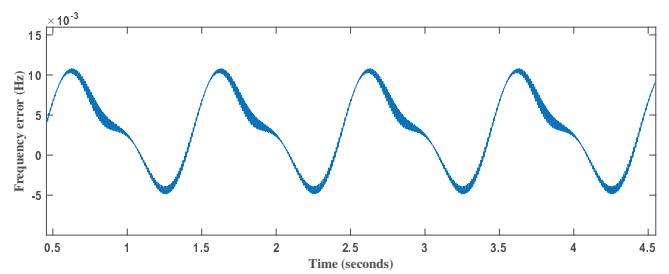

(e) Three cycles

Figure 4.2: Frequency Error in Prony method for DFT based phasor inputs.

The frequency error (absolute value) for the test signal whose phasors are obtained from PLL is provided in Table 4.2 . 


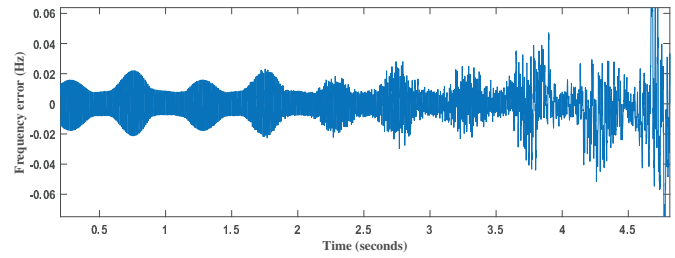

(a) Quarter cycle

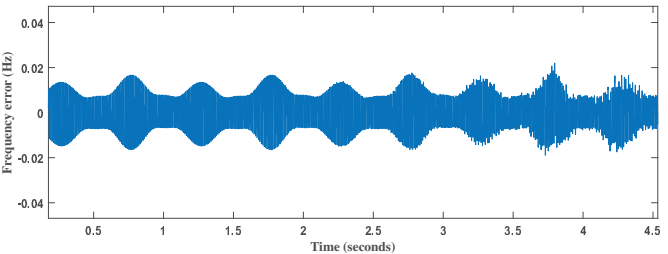

(b) Half cycle

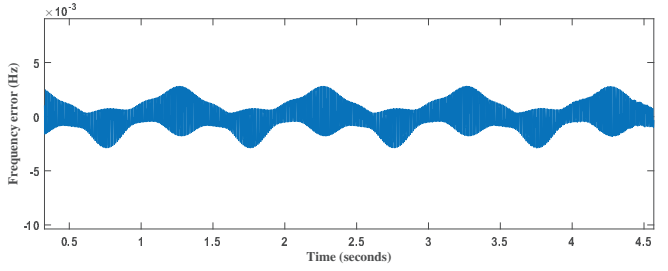

(c) One cycle

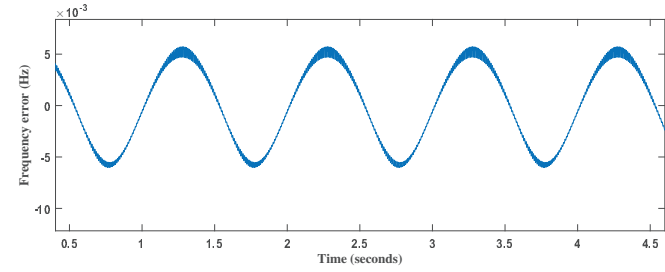

(d) Two cycles

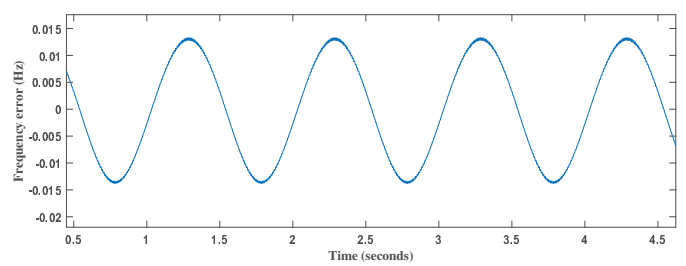

(e) Three cycles

Figure 4.3: Frequency Error in PF method for DFT based phasor inputs.

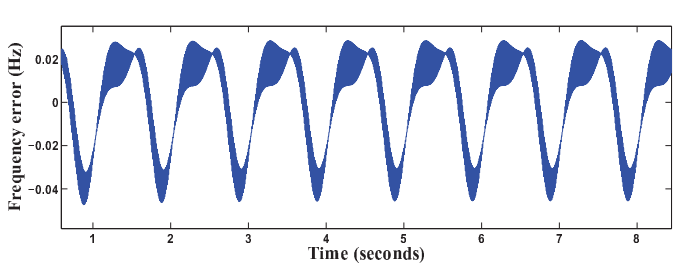

(a) Quarter cycle

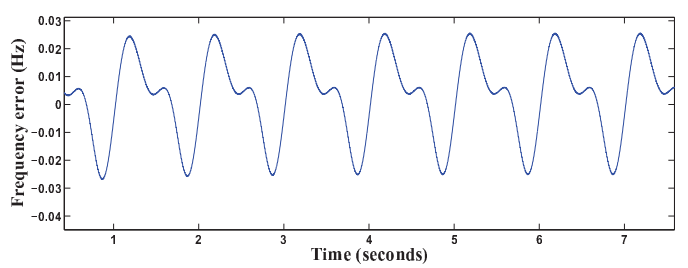

(b) Half cycle

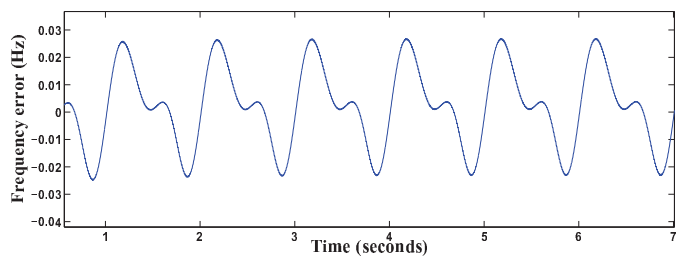

(c) One cycle

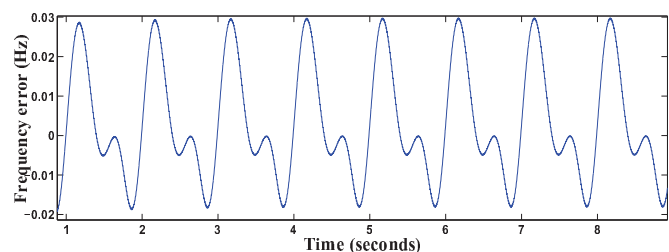

(d) Two cycles

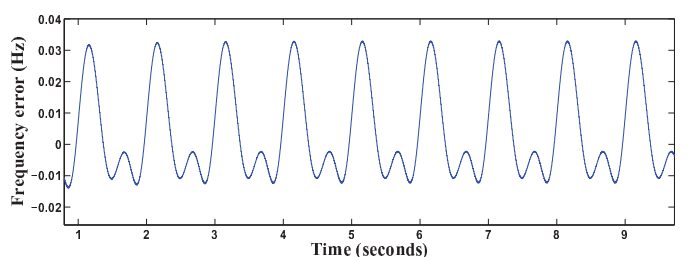

(e) Three cycles

Figure 4.4: Frequency Error in CLS method for PLL based phasor inputs.

From Table 4.2, it can be seen that freqeuncy error is least in the case of CLS method when half cycle window length is considered. When prony method is considered, half cycle window length is 


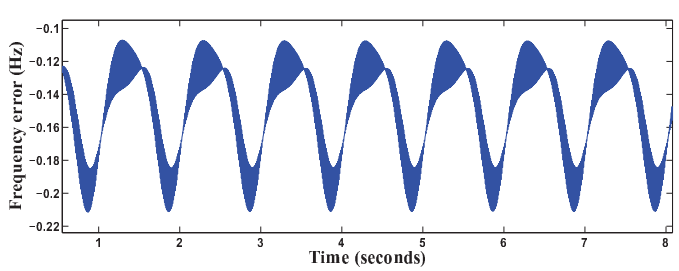

(a) Quarter cycle

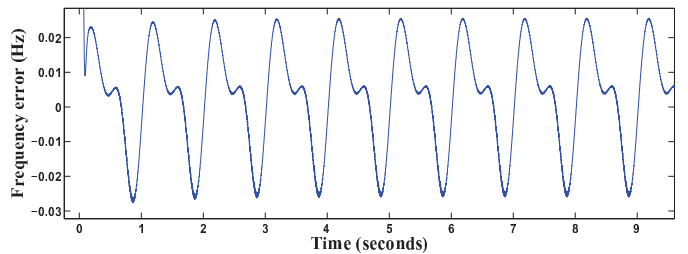

(b) Half cycle

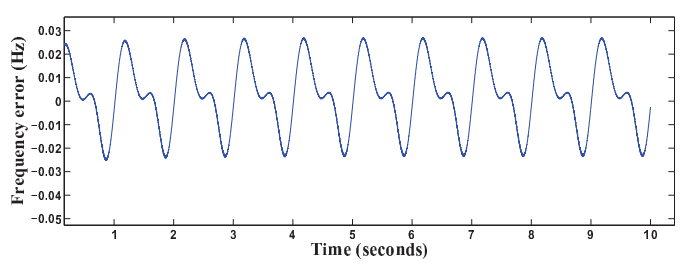

(c) One cycle

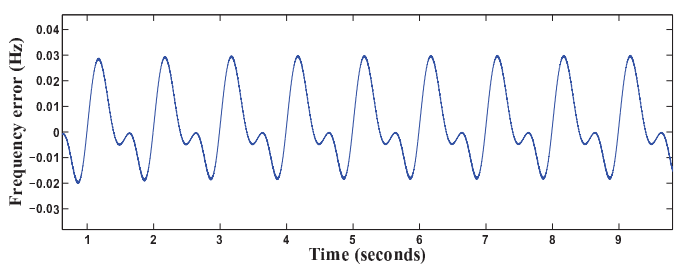

(d) Two cycles

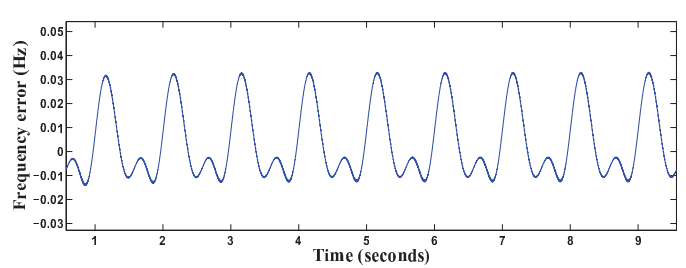

(e) Three cycles

Figure 4.5: Frequency Error in Prony method for PLL based phasor inputs.

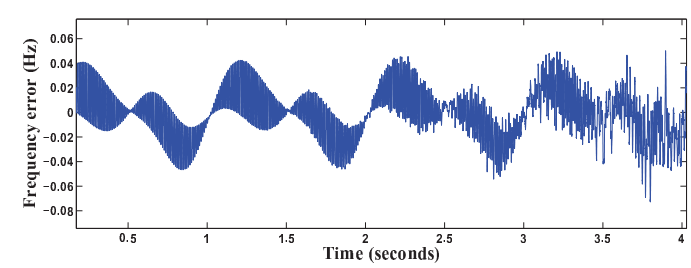

(a) Quarter cycle

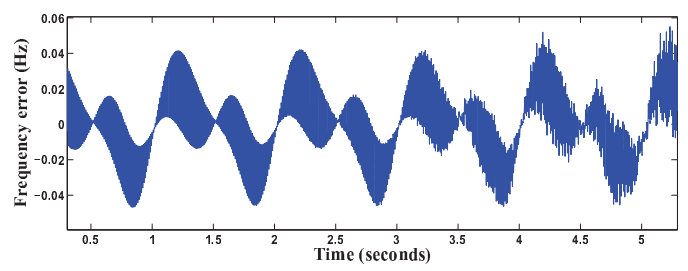

(b) Half cycle

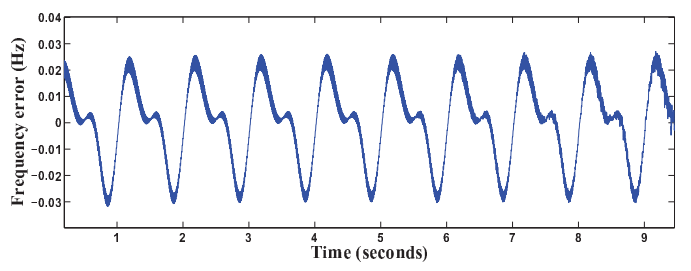

(c) One cycle

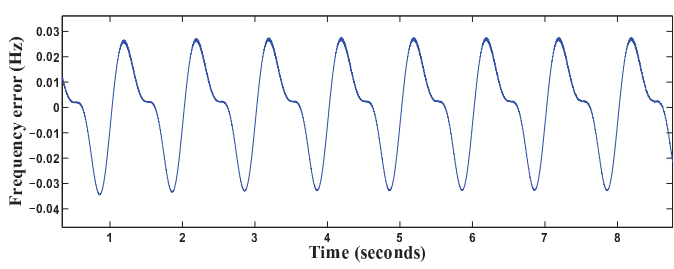

(d) Two cycles

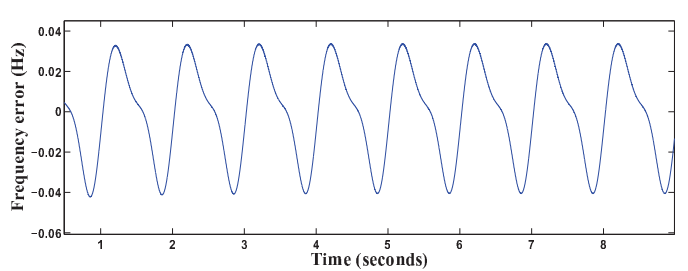

(e) Three cycles

Figure 4.6: Frequency Error in PF method for PLL based phasor inputs.

suggested and the polynomial fitting method one cycle window length is recommended for obtaining 
Table 4.2: Frequency Error $(\mathrm{Hz})$ for Phasors Obtained from PLL

\begin{tabular}{|c|c|c|c|c|c|}
\hline \multirow{2}{*}{ Methods } & \multicolumn{5}{|c|}{ Window length } \\
\cline { 2 - 6 } & QC & HC & OC & TC & THC \\
\hline CLS & 0.046 & 0.025 & 0.0268 & 0.029 & 0.033 \\
\hline Prony & 0.21 & 0.026 & 0.027 & 0.030 & 0.033 \\
\hline PF & 0.050 & 0.046 & 0.030 & 0.033 & 0.040 \\
\hline PLL & \multicolumn{6}{|c|}{0.033} \\
\hline HC-half cycle, OC-one cycle, QC-quarter cycle \\
TC-two cycles, THC-Three cycles \\
\hline
\end{tabular}

minimum frequency error. The errors in frequency estimation by different algorithms with PLLbased phasor input are plotted in Fig 4.4, 4.5, and 4.6.

\subsection{Summary}

Different frequency algorithms are explained in details in this chapter. A comparison between the frequency estimation algorithm is presented with the help of a case study. The dynamically varying system frequency can be accurately estimated from its positive sequence phasor using CLS technique. For frequency estimation in the case of PLL, the phasors obtained from PLL can be used in combination with the frequency estimation such as CLS or prony for better accuracy. 


\section{Chapter 5}

\section{Time Scaling Phenomenon in Phasor Calculation}

\subsection{Introduction}

In existing literature, only the basic signal processing algorithm for phasor measurement are addressed. The algorithm validations are made through off-line simulations by assuming the involvement of only one clock in phasor calculation. A time-scaling phenomenon happens because of the involvement of two different clocks in reality. The time-scaling may lead to inaccuracy in phasor calculation. The time-scaling phenomenon is investigated through a hardware based PMU set-up developed with the help of a real-time simulator. The phasor and the frequency estimation technique considered for the experimental setup are DFT and CLS techniques respectively.

\subsection{Time Scaling}

The time scaling phenomenon happens between two different clocks. The time measured between two events may not be the same in all the clocks. In general, the time measured by one clock is a scaled up or scaled down value of time measured by another clock. That is,

$$
t_{c 1}=p_{s} t_{c 2}
$$

Here, $t_{c 1}$ and $t_{c 2}$ are the time values measured by clock 1 and clock 2 , respectively, with respect to the same time reference instant, and $p_{s}$ is the scaling factor. The reference time instant indicates the moment at which the value of time variable concerned is set to zero. The rate of variation of a quantity depends upon clock that is used. Let, $y$ indicates the rate of variation of quantity $x$. That is,

$$
y=\frac{d x}{d t}
$$


The measured values $y$ with respect to Clock 1 and Clock2 are indicated by $y_{c 1}$ and $y_{c 2}$, respectively. Clearly,

$$
y_{c 1}=\frac{d x}{d t_{c 1}}=\frac{1}{p_{s}} \frac{d x}{d t_{c 2}}=\frac{1}{p_{s}} y_{c 2} .
$$

Therefore, any sort of frequency estimation depends upon the clock used.

For a sinusoidal signal, the phase angle can be defined only with respect to a particular time variable that corresponds to a specific clock and a specific time reference instant. Consider the following sinusoidal signal,

$$
v\left(t_{c 1}\right)=V_{m} \cos \left(\omega_{0} t_{c 1}+\phi_{1}\right) .
$$

The above signal can be alternatively written as,

$$
\begin{aligned}
v\left(t_{c 2}\right) & =V_{m} \cos \left(\omega_{0} p_{s} t_{c 2}+\phi_{1}\right) \\
& =V_{m} \cos \left(\omega_{0} t_{c 2}+\left(p_{s}-1\right) \omega_{0} t_{c 2}+\phi_{1}\right) .
\end{aligned}
$$

Therefore, the phase angles estimated with respect to time variables $t_{c 1}$ and $t_{c 2}$ (indicated by $\phi_{c 1}$ and $\phi_{c 2}$, respectively) would be found as follows.

$$
\begin{aligned}
\phi_{c 1} & =\phi_{1} \\
\phi_{c 2} & =\phi_{1}+\left(p_{s}-1\right) \omega_{0} t_{c 2} .
\end{aligned}
$$

Clearly, even though the phase angle may be found to be constant with respect to one clock, it may be seen varying with respect to another clock.

\subsection{Time Scaling Phenomenon in PMU}

The complete organization of PMU is shown in Fig. 5.1. There are two different clocks that are involved in phasor calculation. The first one is the GPS clock and the second one is the microprocessor clock. The phasor calculation is carried out in two steps. At first, the phasors is calculated with respect to a local time reference that is specified to each PMU. Subsequently, the locally referred phasor is converted to the globally referred phasor through appropriate phase shifting. The standard global or common reference is $00: 00: 00$, January 1, 1970, according to the GPS clock [2]. The locally referred phasor is the first calculated with respect to the microprocessor clock according to the following equation,

$$
\bar{V}_{T_{c 2}}[n]=\frac{2}{N} \sum_{r=M}^{N+M-1} v[n+r] e^{-j(m+r) \frac{2 \pi}{N}}
$$

Here in $T_{c 2}$, the subscript indicates the microprocessor clock time at the local time reference with respect to the time instant that is taken as the global reference. The time quantity $T_{c 2}$ can be 


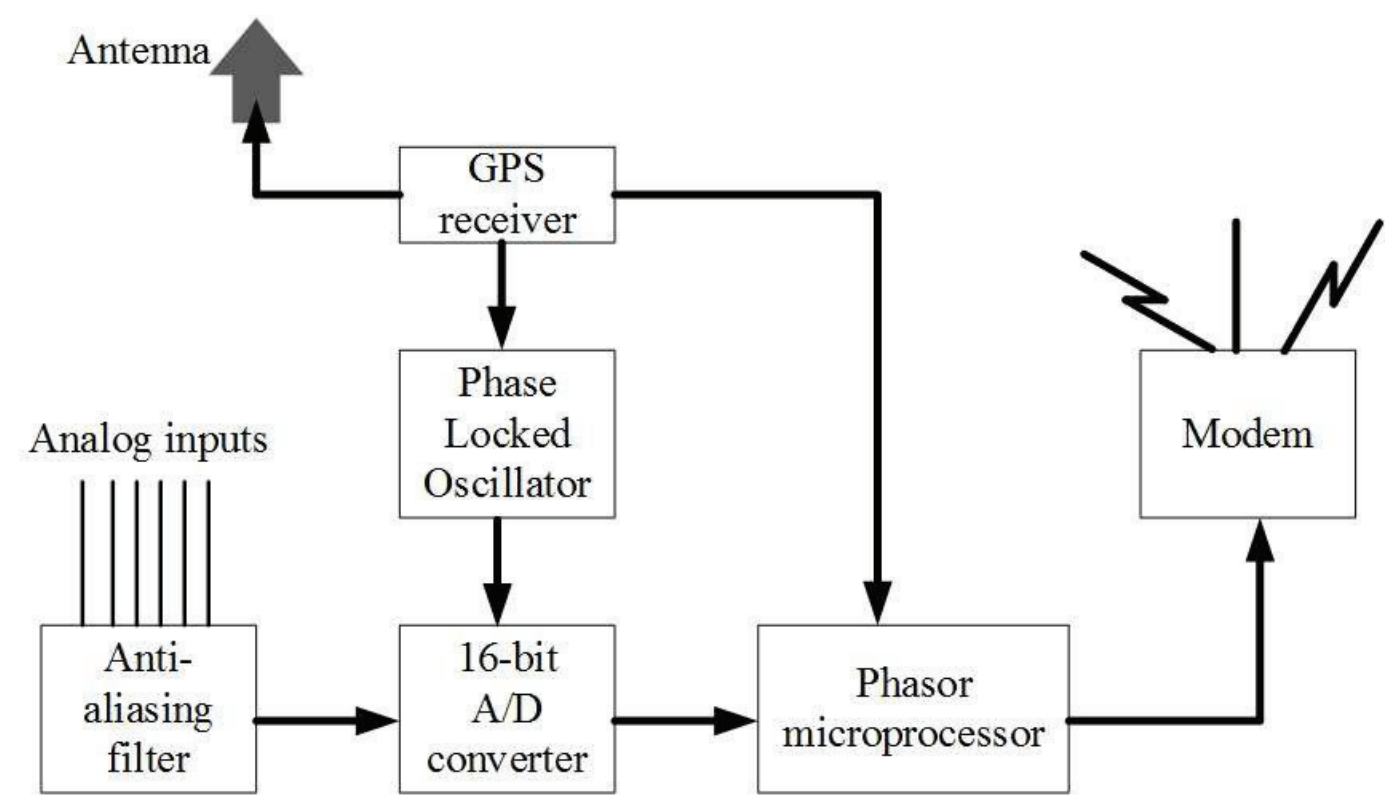

Figure 5.1: PMU Block Diagram.

further be expressed as follows,

$$
T_{c 2}=T_{0, c 2}+\Delta T_{c 2}
$$

Where, $T_{0, c 2}$ is the microprocessor clock time at the instant when the PMU starts sampling. The sampling index is counted from $t_{c 2}=T_{0, c 2}$. That is, the $(n+r)$ th sample corresponds to the time instant $t_{c 2}=T_{0, c 2}+(n+r) T_{s}$. The value of $m$ is obtained from the following equation.

$$
T_{0, c 2}+(n+r) T_{s}=T_{0, c 2}+\Delta T_{c 2}+(m+r) T_{s} .
$$

In case the static local time reference, $\Delta T_{c 2}$ is set fixed to zero. This in turn makes $m$ and $n$ equal. Alternatively, the local time reference can be dynamically varied according to the equation $\Delta T_{c 2}=n T_{s}$ so as to keep $\mathrm{m}$ constant at zero.

In principle, the locally referred phasor calculated with respect to the microprocessor clock is to be first redefined with respect to the GPS clock. From (5.9), the particular conversion formula appears as follows,

$$
\bar{V}_{T_{c 1}[n]}=\bar{V}_{T_{c 2}}[n] e^{-j \omega_{0}\left(p_{s}-1\right) m T_{s}} .
$$

Here in $T_{c 1}$, the subscript indicates the GPS clock time at the local time reference. The final phasor (symbolized as $\bar{V}_{c 1}$ ) with respect to the global reference and GPS clock is obtained through the following phase shifting,

$$
\bar{V}_{c 1}[n]=\bar{V}_{T_{c 1}}[n] e^{j \omega_{0} T_{c 1}}
$$

In reality, it is difficult to precisely determine the value of time scaling factor. Therefore, the following 
formula is practically used to determine the final phasor by assuming the scaling factor to be 1 .

$$
\bar{V}_{c 1, c a l}[n]=\bar{V}_{T_{c 2}[n] e^{j \omega_{0} T_{c 1}}} .
$$

From (5.12),(5.13), and (5.14), we get,

$$
\bar{V}_{c 1, c a l}[n]=\bar{V}_{c 1}[n] e^{j \omega_{0}\left(p_{s}-1\right) m T_{s}} .
$$

In case of static local time reference, $m$ continuously grows with $\mathrm{n}$. This, in long run, may introduce significant phase angle error. On the other hand, no phase angle error is introduced because of time scaling phenomenon for the dynamically varied local time reference since $m$ is always maintained at zero.

From the above discussion, it can be concluded that the choice of dynamic time reference is better so as to ensure required accuracy in phasor calculation. However, the dynamic time reference based phasor calculation is often criticized abouts its computational time requirement. There is, in fact, no recursive form produced in literature for the dynamic time reference based phasor calculation, although the same is available when the time reference is kept static. Therefore, the computationally efficiency of dynamic time reference based phasor calculation remained undoubtful. In reality, a generalized recursive form is possible for updating the phasor calculation with respect to any arbitary choice of time reference. From (5.9),

$$
\begin{gathered}
\bar{V}_{T_{c 2}}[n+1]=\frac{2}{N} \sum_{r=M}^{N-1+M} v[n+1+r] e^{-j(m+r) \frac{2 \pi}{N}} \\
\bar{V}_{T_{c 2}}[n+1]=\frac{2}{N} \sum_{r=M-1}^{N-2+M} v[n+1+r] e^{-j(m+r) \frac{2 \pi}{N}} \\
+\frac{2}{N}\left\{v[N+M+n] e^{-j(M-1+m+N) \frac{2 \pi}{N}}-v[M+n] e^{-j(M-1+m) \frac{2 \pi}{N}}\right\} \\
\bar{V}_{T_{c 2}}[n+1]=\frac{2}{N} \sum_{r=M-1}^{N-2+M} v[n+1+r] e^{-j(m+r) \frac{2 \pi}{N}} \\
+\frac{2}{N}\{v[N+M+n]-v[M+n]\} e^{-j(M-1+m) \frac{2 \pi}{N}} \\
\bar{V}_{T_{c 2}[n+1]}=\bar{V}_{T_{c 2}[n]+\frac{2}{N}\{v[N+M+n]-v[M+n]\} e^{-j(M-1+m) \frac{2 \pi}{N}}}
\end{gathered}
$$

Therefore, there is there is no true computational complexity in the dynamic time reference based phasor calculation. 


\subsection{Experimental Setup}

The experimental setup consists of an eMEGAsim [24] simulator, host computer, and screw terminals. The eMEGAsim is a specialized computing facility used for data acquisition and real-time simulation of dynamical systems. The eMEGAsim simulator used consists of two target computers (Target 1 and Target 2). The data is exchanged between the targets through analog I/O ports. The maximum value that can be passed between these I/O ports is between -16 to 16 . If the values used are greater than these values, they must be scaled down at the sending port and must be scaled up at the receiving port. The time signals (for time-stamping and time reference synchronization) are generated from Target 1 . The test three-phase AC signal is also generated from Target 1 . The phasor and frequency calculation algorithms are run in Target 2. Target 2 also creates the timestamped phasor data packet and communicates it to the host computer via Ethernet port. Target 1 and Target 2 are run independently and asynchronously. Target 1 and Target 2 are run at time steps of 10 microsecond (that is the minimum permissible time step for eMEGAsim simulaor) and 200 microsecond respectively. Therefore, the time signals could be generated only at the interval of 10 microsecond. Target 1 is started first. The time instant at which Target 2 starts up is noted down by Target 1 (according to its own clock) through a start pulse from Target 2 . The schematic diagram of the experimental setup is shown in Fig. 5.2. The models required for simulation are developed in MATLAB/Simulink.

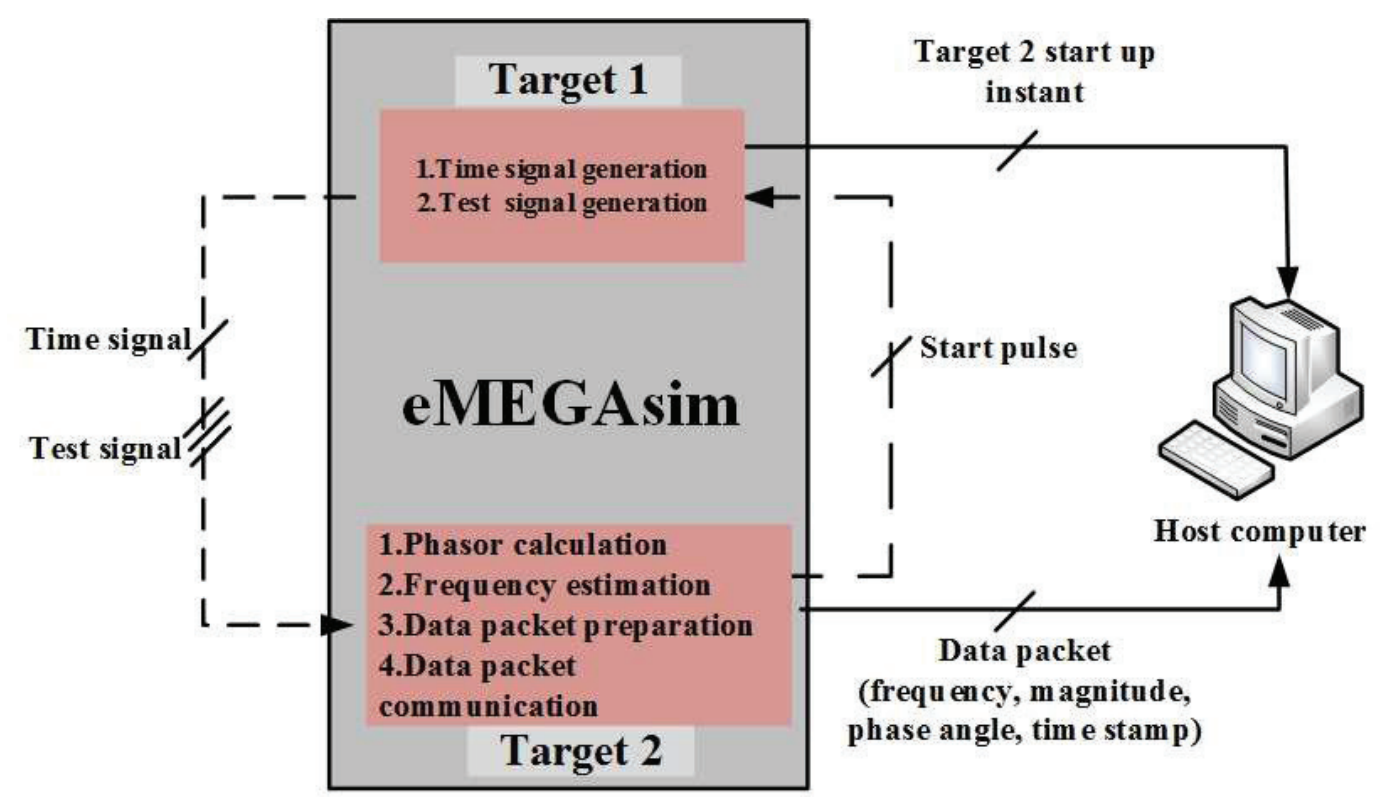

Figure 5.2: Experimental setup using eMEGAsim.

The time instant for the phasor and frequency measurement is obtained using a counter based approach. Two free running counters are placed in Target 1 one with a sampling time interval same as Target $1(10 \mu \mathrm{s})$ and the other with a higher sampling time interval of $1 \mathrm{~ms}$. The fast free running counter placed in Target 1 is used for time signal generation and its count value is set from 0 to 99 . The slow free running counter placed in Target 1 is used for providing the time reference synchronization and is set to a very high value. The slow free running counter is also used for storing the count value of the time instant at which Target 2 is started. The time instant that 
is calculated corresponding to the count value stored is with respect to Target 1 clock. An event triggered counter is placed in Target 2, it is incremented every time when the fast free running counter placed in Target 1 completes one full cycle count. The event triggered counter placed in Target 2 is replica of slow free running counter placed in Target 1 as there is a hardware limitation in passing the value from Target 1 to Target 2 . The count value obtained from the fast free running counter placed in Target 1 and the event triggered counter placed in Target 2 are added and is multiplied with the sampling time interval of Target 1 to obtain the time stamping for the estimated phasor and frequency quantities. With the help of the stored counter value at the time instant at which Target 2 is started, the estimated phasor and frequency quantities can be time stamped with respect to the Target 1 clock time reference.

The experimental results obtained for the three phase signal provided in Table 3.1 are shown below. The signals are tested with two different frequency. The time scaling phenomenon in particular affects the phase angle measurement. The amplitude plot of the signal is plotted in Fig. 5.3. The effect of time scaling phenomenon in phase angle for two different frequency is discussed in the

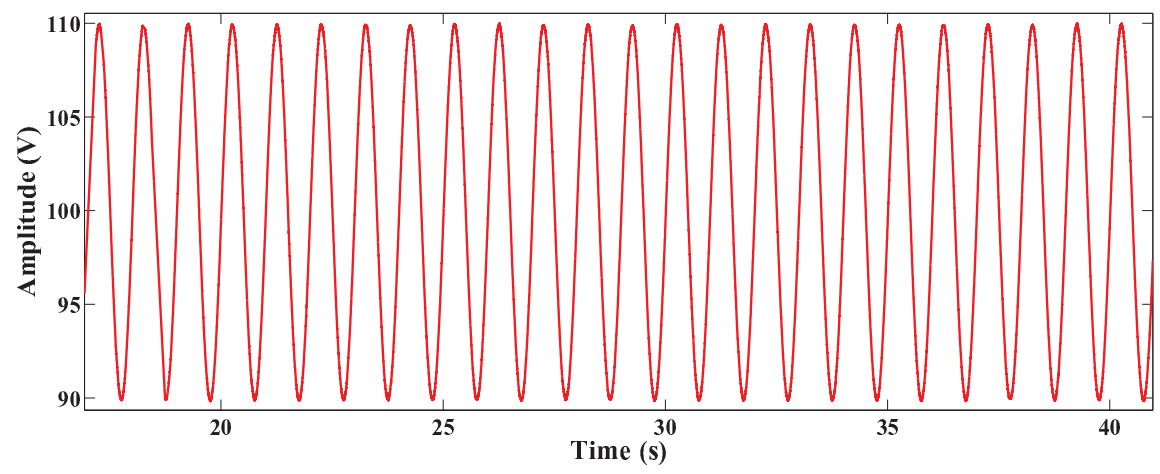

Figure 5.3: Amplitude Plot.

following section.

\subsubsection{Constant frequency}

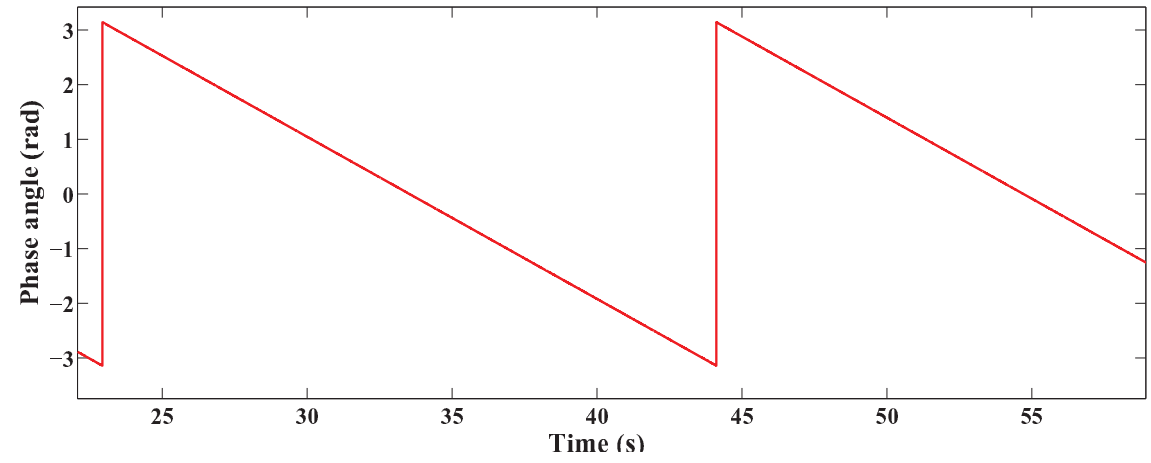

Figure 5.4: Phase angle Without Correction.

In this case, a the system frequency is considered to be at the nominal value $(50 \mathrm{~Hz})$. The phase 


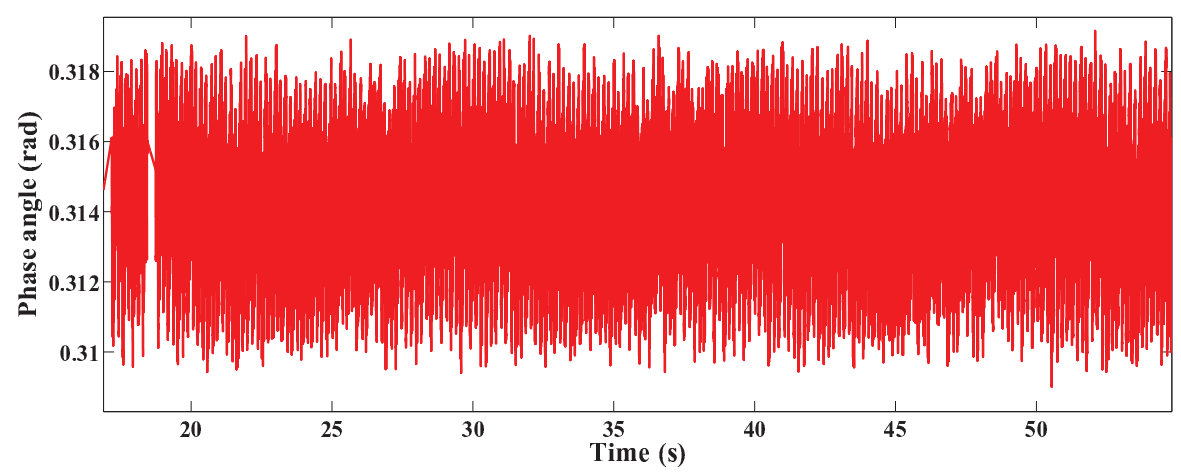

Figure 5.5: Phase Angle referred to Target 1 time reference from the start instant of Target 2.

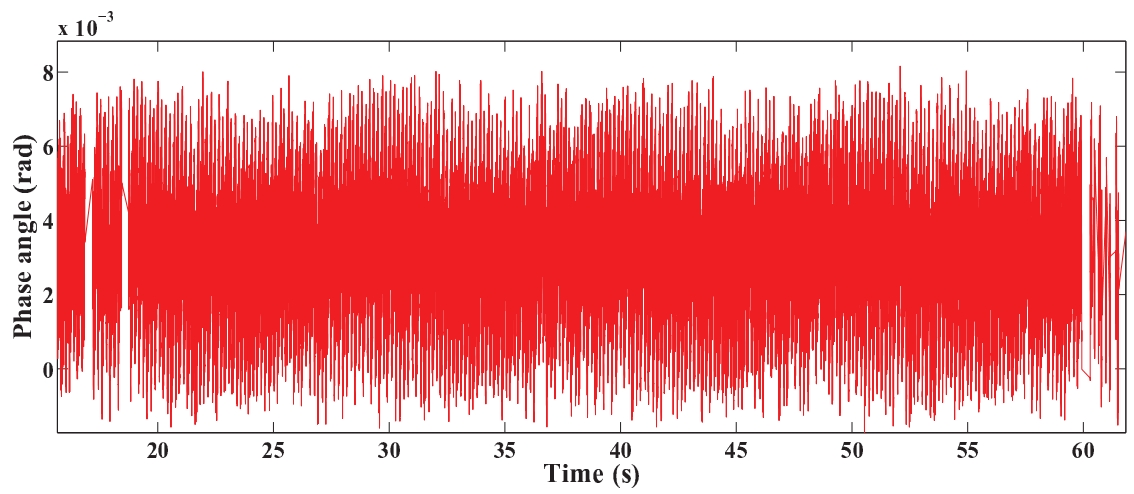

Figure 5.6: Phase Angle referred to Target 1 Start Instant.

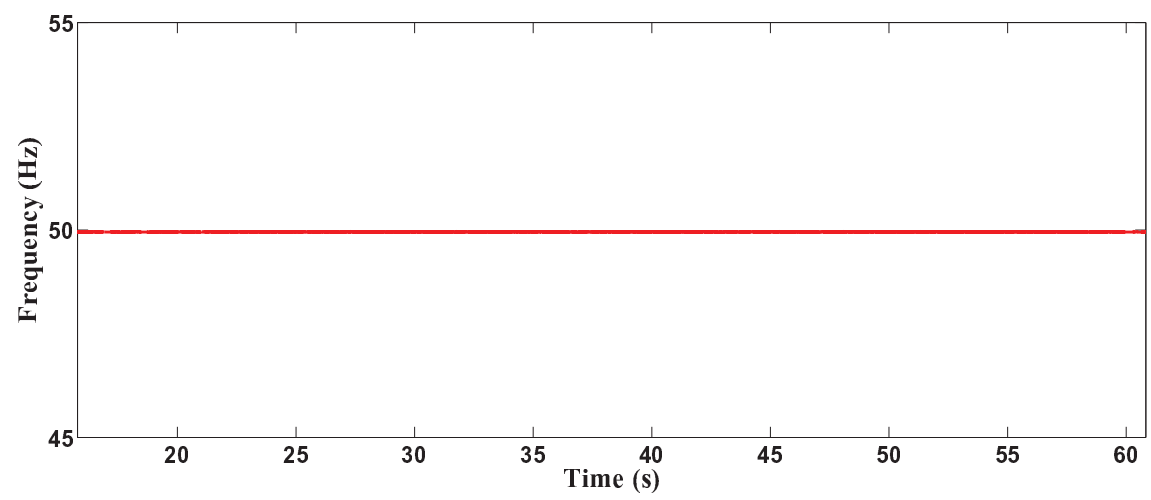

Figure 5.7: Frequency Plot $(50 \mathrm{~Hz})$.

angle of the sampled signal obtained from Target 1 without any angle correction is provided in Fig. 5.4. The phase angle plot with respect to Target 1 time reference from instant at which Target 2 is started is plotted in Fig. 5.5. The phase angle plot is also provided with scaling factor correction. The slope found in Fig. 5.4 i not found in Fig. 5.5. Finally, the phase angle plot with respect start instant of $\mathrm{T} 1$ is plotted in Fig. 5.6. It is done with help of the count value stored at the time instant at which Target 2 is started. The frequency plot of the signal with respect to Target 1 time reference is plotted in Fig. 5.7. 


\subsubsection{Sinusoidally Varying Frequency}

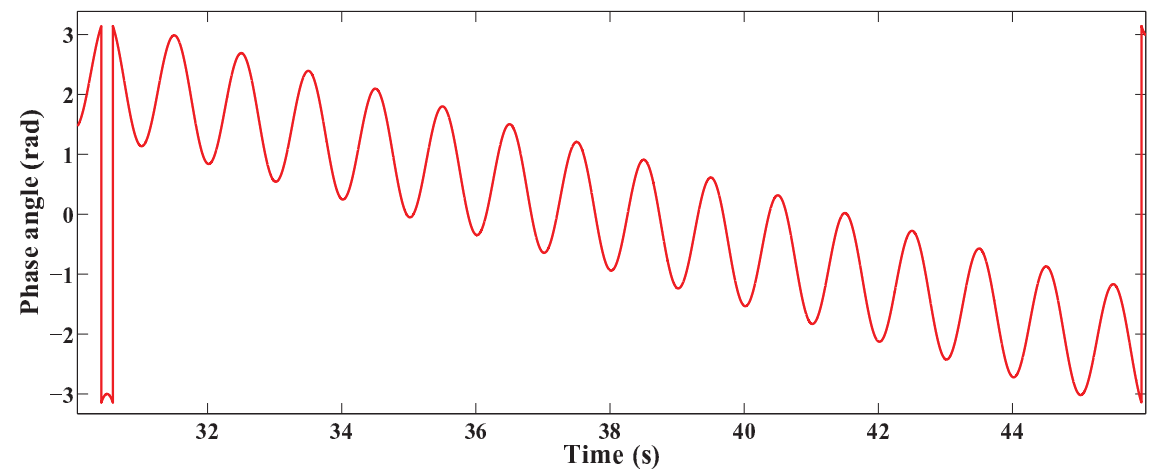

Figure 5.8: Phase angle Without Correction.

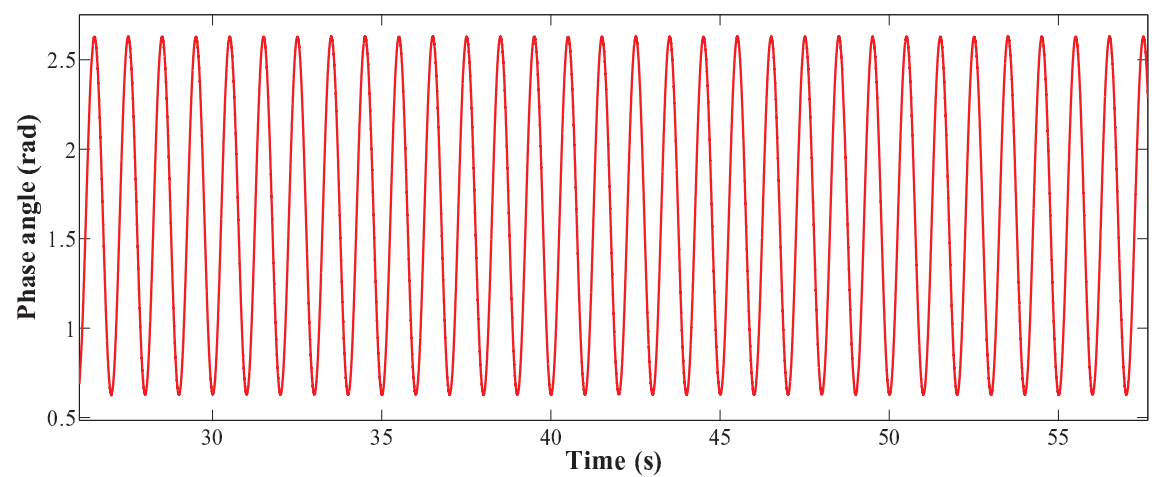

Figure 5.9: Phase Angle referred to Target 1 time reference from the start instant of Target 2.

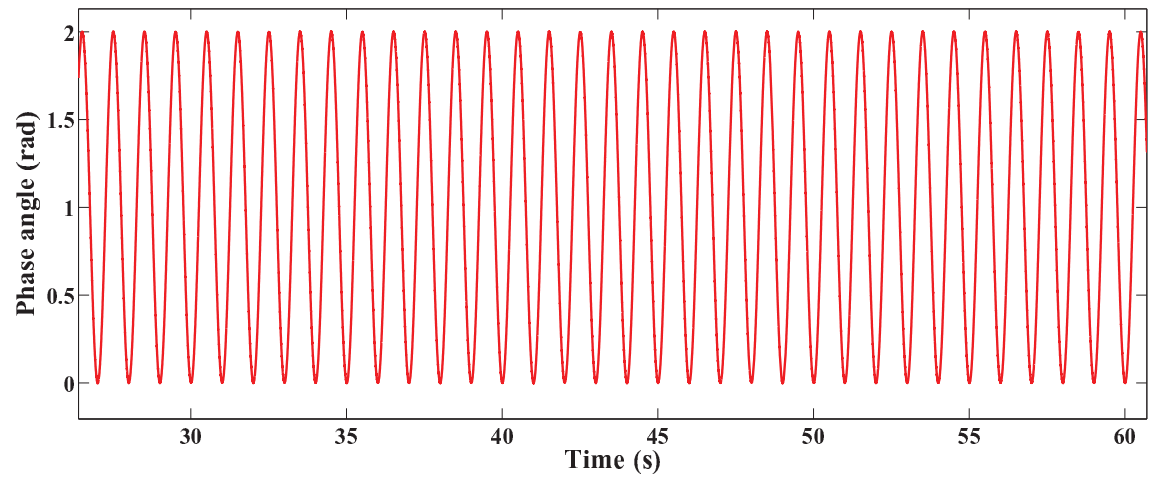

Figure 5.10: Phase Angle referred to Target 1 Start Instant.

In this case, the system frequency is slowly varying with time.

$$
f=50+\sin (2 \pi t) .
$$

The phase angle of the sampled signal obtained from Target 1 without any angle correction is 


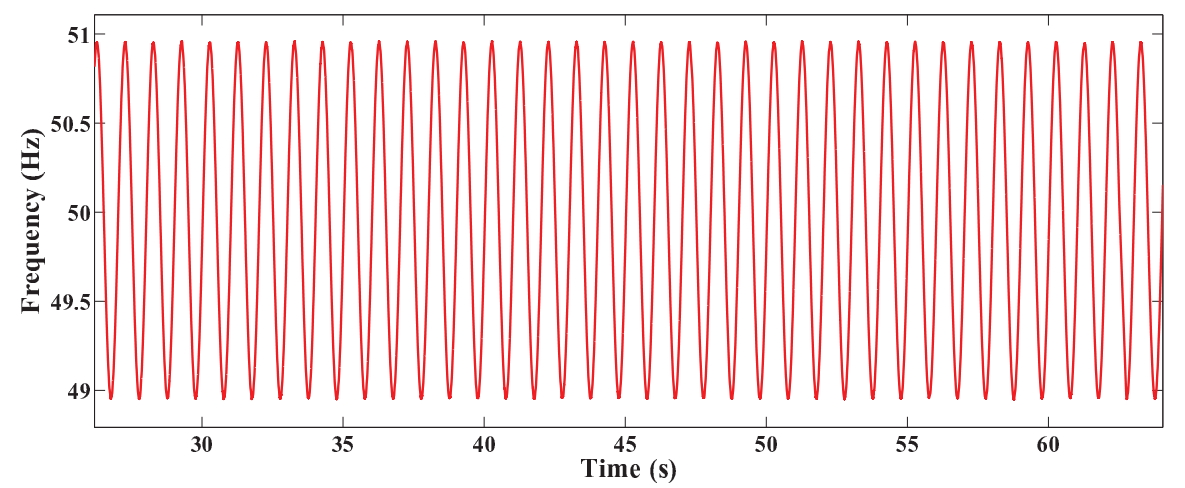

Figure 5.11: Frequency Plot $((50+\sin (2 \pi t)) \mathrm{Hz})$.

provided in Fig. 5.8. The phase angle plot with respect to Target 1 time reference from instant at which Target 2 is started is plotted in Fig. 5.9. The phase angle plot is also provided with scaling factor correction. Finally, the phase angle plot with respect start instant of T1 is plotted in Fig. 5.10. It is done with help of the count value stored at the time instant at which Target 2 is started. The frequency plot of the signal with respect to Target 1 time reference is plotted in Fig. 5.11.

\subsection{Summary}

The concept of time scaling is discussed and its effects in phasor estimation is also explained. The time scaling phenomenon does not affect the phasor measurement when dynamic time reference is used. The time stamping of the estimated phasor and frequency is performed through HIL simulation. The time stamping provided is similar to the PMU time stamping but local time reference is used in this case and the experimental results suggests that the time stamping provided is found to be quite accurate. 


\section{Chapter 6}

\section{Conclusion and Future Work}

\subsection{Conclusion}

A comparative study on different phasor and frequency measurement techniques that are available in the power systems is presented in this thesis. From the simulation study it can observed that the DFT based phasor estimation techniques provide better accuracy compared to PLL even when the signal parameters are varying. The CLS technique is found to be more accurate compared to other frequency estimation techniques considered for study. For improving the accuracy of estimated frequency in the case of PLL, the phasors obtained from PLL can be used in combination with CLS or prony algorithm. Finally, the simulation study performed is helpful in realizing the ascendancy amongst the different phasor and frequency estimation algorithms and its applicability for real-time measurements.

The HIL simulation performed using eMEGAsim for estimating phasor and frequency provides an overview of time scaling phenomenon and its effects on phase angle and frequency measurement. It is found out that the use of dynamic time reference for phasor and frequency measurement proves to be superior compared to the the static time reference after investigating the time scaling phenomenon.

\subsection{Future Work}

- Hardware implementation of frequency and phasor estimation algorithms using Digital Signal Processors.

- Laboratory development of an phasor measurement unit in compliance with the synchrophasor standard. 


\section{References}

[1] [Online]. Available: http://www.electricalindia.in/blog/post/id/8522/phasor-measurementunits-for-power-systems.

[2] IEEE Standard for Synchrophasor Measurements for Power System, in IEEE Std C37.118.12011 (Revision of IEEE Std C37.118-2005), Dec. 2011.

[3] B. Gou, "Optimal Placement of PMUs by Integer Linear Programming," in IEEE Trans. Power Sys., vol. 23, no. 3, pp. 1525 - 1526, Aug. 2008.

[4] J. Ren and M. Kezunovic, "Real time power system frequency and phasor estimation scheme using recursive wavelet transform," IEEE Trans. Power Del., vol. 26, no. 3, pp. $1392-1402$, Jul. 2011.

[5] V. Backmutsky and V. Zmudikov, "Accurate frequency estimation in power systems by DSP," in Proc. 18th Conv., Elect. Electron. Engineers, May 1995, pp. $712-715$.

[6] M. M. Begovic, M. M. Djuric, S. Dunlap, and A. G. Phadke, "Frequency tracking in power networks in the presence of harmonics," IEEE Trans. Power Del., vol. 8, no. 2, pp. $480-486$, Apr. 1993.

[7] A. K. Pradhan, A. Routray, and A. Basak, "Power system frequency estimation using least mean square technique," IEEE Trans. Power Del., vol. 20, no. 3, pp. 1812 - 1816, Jul. 2005.

[8] P. K. Dash, A. K. Pradhan, and G. Panda, "Frequency estimation of distorted power system signals using extended complex Kalman filter," IEEE Trans. Power Del., vol. 14, no. 3, pp. $761-766$, Jul. 1999.

[9] V. V. Terzija, M. B. Djuric, and B. D. Kovacevic, "Voltage phasor and local system frequency estimation using Newton type algorithm," IEEE Trans. Power Del., vol. 9, no. 3, pp. 1368-1374, Jul. 1994.

[10] R. K. Mai, Z. Y. He, L. Fu, W.He, and Z. Q. Bo, "Dynamic phasor and frequency estimator for phasor measurement units," in IET Gen. Trans. Distri., vol. 4, no.1, pp. 73-83, Jan. 2010.

[11] C. D. L. da Silva, G. Cardoso Junior, L. Mariotto, and G. Marchesan, "Phasor estimation in power system using a neural network with online training for numerical relays purposes," in IET Sci. Meas. Technol. , vol. 9, no. 7, pp. 836 - 841, Oct. 2015. 
[12] A. Ashrafian and M. Mirsalim, "On-line recursive method of phasor and frequency estimation for power system monitoring and relaying," in IET Gen. Trans. Distri., vol. 10, no. 8, pp. 2002 - 2011, May 2016.

[13] T. Lin and A. D. Jun, "Novel complex filter with recursive algorithm for phasor computation in power-quality monitoring," in IEE Proc. Gen. Trans. Distri., vol. 153, no. 3, pp. $283-290$, May 2006.

[14] L. Yang, Z. Jiao, X. Kang, G. Song and J. Suonan, "Fast algorithm for estimating power frequency phasors under power system transients," in IET Gen. Trans. Distri., vol. 9, no. 4, pp. 395 - 403, May 2015.

[15] T. Lobos and J. Rezmer, "Real-time determination of power system frequency," IEEE Trans. Instrum. Meas., vol. 46, no. 4, pp. 877 - 881, Aug. 1997.

[16] Y. Xia, Y. He, and K. Wang, "A complex least squares enhanced smart DFT technique for power system frequency estimation," IEEE Trans. Power Del., vol. PP, no. 99, pp. 1 - 1, doi: 10.1109/TPWRD.2015.2418778.

[17] A. G. Phadke and J. S. Thorp, Synchronized phasor measurements and their applications, New York: Springer, 2008.

[18] J. Z. Yang and C. W. Liu, "A precise calculation of power system frequency," IEEE Trans. Power Del., vol. 16, no. 3, pp. 361 - 366, Jul. 2001.

[19] P. K. Das, K. R. Krishnanand, and M. Padhee, "Fast recursive Gauss-Newton adaptive filter for the estimation of power system frequency and harmonics in a noisy enviornment," IET Gen. Trans. Distri., vol. 5, no. 12, pp. 1277 - 1289, Dec. 2011.

[20] H. S. Zhao, Z. S. Liu, G. W. Song, and D. L. Wei, "The analysis of frequency deviation on synchrophasor calculation and correction methods," in Proc. Int. Conf. Sustainable Power Gen. Supply, 2009, pp. $1-5$.

[21] G. Benmouyal, "An adaptive sampling-interval generator for digital relaying," in IEEE Trans. Power Del., vol. 4, no. 3, pp. 1602 - 1609, Jul 1989.

[22] D. Hart, D. Novosel, Yi Hu, B. Smith and M. Egolf, "A new frequency tracking and phasor estimation algorithm for generator protection," in IEEE Trans. Power Del., vol. 12, no. 3, pp. 1064 - 1073, Jul. 1997.

[23] R. Chudamani, Krishna Vasudevan, and C. S. Ramalingam, "Real-time estimation of power system frequency using nonlinear least square," IEEE Trans. Power Del., vol. 24, no. 3, pp. 1021 - 1028, Jul. 2009.

[24] [Online]. Available: http://www.opal-rt.com/product/emegaasim-powergird-real-time-digitalhardware-in-the-loop-simulator. 